\title{
MicroRNA-668 represses MTP18 to preserve mitochondrial dynamics in ischemic acute kidney injury
}

\author{
Qingqing Wei, ${ }^{1}$ Haipeng Sun, ${ }^{2}$ Shuwei Song, ${ }^{2}$ Yong Liu, ${ }^{3}$ Pengyuan Liu, ${ }^{3}$ Man Jiang Livingston, ${ }^{1}$ Jianwen Wang, ${ }^{4}$ Mingyu Liang, ${ }^{3}$ \\ Qing-Sheng Mi, ${ }^{5}$ Yuqing Huo, ${ }^{6}$ Norris Stanley Nahman, ${ }^{7}$ Changlin Mei, ${ }^{2}$ and Zheng Dong ${ }^{1,8,9}$ \\ 'Department of Cellular Biology and Anatomy, Medical College of Ceorgia at Augusta University, Augusta, Georgia, USA. Department of Nephrology, Changzheng Hospital, Second Military Medical University, \\ Shanghai, China. ${ }^{3}$ Department of Physiology, Center of Systems Molecular Medicine, Medical College of Wisconsin, Milwaukee, Wisconsin, USA. ${ }^{4}$ Department of Nephrology, Third Xiangya Hospital, \\ Central South University, Changsha, China. ${ }^{5}$ Center for Cutaneous Biology and Immunology Research, Department of Dermatology, Henry Ford Health System, Detroit, Michigan, USA. ${ }^{6}$ Vascular Biology \\ Center and 'Department of Medicine, Medical College of Ceorgia at Augusta University, Augusta, Georgia, USA. ${ }^{8}$ Department of Nephrology, Second Xiangya Hospital, Central South University, \\ Changsha, China. ${ }^{9}$ Charlie Norwood VA Medical Center, Augusta, Ceorgia, USA
}

\begin{abstract}
The pathogenesis of ischemic diseases remains unclear. Here we demonstrate the induction of microRNA-668 (miR-668) in ischemic acute kidney injury (AKI) in human patients, mice, and renal tubular cells. The induction was HIF-1 dependent, as HIF-1 deficiency in cells and kidney proximal tubules attenuated miR-668 expression. We further identified a functional HIF-1 binding site in the miR-668 gene promoter. Anti-miR-668 increased apoptosis in renal tubular cells and enhanced ischemic AKI in mice, whereas miR-668 mimic was protective. Mechanistically, anti-miR-668 induced mitochondrial fragmentation, whereas miR-668 blocked mitochondrial fragmentation during hypoxia. We analyzed miR-668 target genes through immunoprecipitation of microRNA-induced silencing complexes followed by RNA deep sequencing and identified 124 protein-coding genes as likely targets of miR-668. Among these genes, only mitochondrial protein 18 kDa (MTP18) has been implicated in mitochondrial dynamics. In renal cells and mouse kidneys, miR-668 mimic suppressed MTP18, whereas antimiR-668 increased MTP18 expression. Luciferase microRNA target reporter assay further verified MTP18 as a direct target of miR-668. In renal tubular cells, knockdown of MTP18 suppressed mitochondrial fragmentation and apoptosis. Together, the results suggest that miR-668 is induced via HIF-1 in ischemic AKI and that, upon induction, miR-668 represses MTP18 to preserve mitochondrial dynamics for renal tubular cell survival and kidney protection.
\end{abstract}

\section{Introduction}

Tissue damage by ischemia/reperfusion leads to the development of a major class of disease, called ischemic disease, including myocardial infarction, stroke, shock liver, and acute kidney injury (AKI). Despite decades of research, the cellular and molecular basis of ischemic disease remains unclear, and effective therapies are still lacking (1-3). In this regard, mitochondrial damage has been implicated in the pathogenesis of ischemic disease for a long time. The research in recent years has further demonstrated an important role of the disruption of mitochondrial dynamics in ischemic cell injury and death. In kidneys, ischemic AKI is associated with the disruption of mitochondrial dynamics resulting in mitochondrial fragmentation, and, notably, inhibition of mitochondrial fission or fragmentation can prevent kidney damage, cell death, and functional decline of AKI $(4,5)$. Mitochondrial dynamics is also perturbed in nephrotoxic and septic AKI (4-7). Similar findings have been reported in ischemia/reperfusion

\section{Related Commentary: p. 5216}

Conflict of interest: The authors have declared that no conflict of interest exists. License: Copyright 2018, American Society for Clinical Investigation. Submitted: April 25, 2018; Accepted: October 4, 2018.

Reference information: J Clin Invest. 2018;128(12):5448-5464. https://doi.org/10.1172/JCl121859. injury of the heart and brain $(8,9)$. Interestingly, mitochondrial fragmentation also contributes to the pathogenesis of chronic or degenerative diseases, such as Huntington's disease (10) and diabetic nephropathy $(11,12)$. The latest work by Perry et al. has further suggested a role of mitochondrial dynamics in kidney repair following AKI (13).

Mitochondrial dynamics is the homeostatic balance between mitochondrial fission and fusion, which are governed by 2 separate classes of proteins $(14,15)$. Mitochondrial fusion involves mitofusins 1 and 2 at the mitochondrial outer membrane and optic atrophy 1 (OPA1) at the mitochondrial inner membrane, whereas Dynamin-related protein 1 (Drp1) is known to be the key protein for mitochondrial fission. In addition to Drp1, other proteins have been implicated in mitochondrial fission, including mitochondrial protein $18 \mathrm{kDa}$ (MTP18) (16, 17), a mitochondrial fission protein examined in the present study. Under pathogenic conditions of cell stress, such as ischemic AKI, Drp1 is dephosphorylated and activated to promote mitochondrial fission (18), while mitofusins and OPA1 are inactivated by protein interactions and proteolytic cleavage, respectively, resulting in the arrest of mitochondrial fusion $(19,20)$. As such, posttranslational modifications, including de/phosphorylation, sumoylation, and proteolytic processing, are recognized as pivotal mechanisms for the regulation of mitochondrial dynamics. Nonetheless, recent work has suggested the regulation of mitochondrial fission and fusion proteins by specific 
microRNAs (21-23), unveiling a new layer of regulatory mechanisms for mitochondrial dynamics.

MicroRNAs are small, noncoding RNAs of 21-25 nucleotides that play important roles in the regulation of gene expression by repressing target gene translation and/or inducing target gene mRNA degradation. In 2010, we reported the mouse model with specific ablation of Dicer (an enzyme for microRNA biogenesis) from kidney proximal tubules (24). These mice were deprived of microRNAs in proximal tubule cells as expected, and, remarkably, they became resistant to ischemic AKI, suggesting an important pathogenic role of microRNAs (24). We and others further identified the microRNA species with significant changes in expression during ischemic AKI $(24,25)$. More recent studies have examined the role and regulation of some of these microRNAs in ischemic AKI, including miR-494, -21, -126, -687, -150, -489, and -17-5p (26-39). Interestingly, some of the microRNAs promote ischemic AKI, but others play renoprotective roles $(32,36,40)$. Despite these findings, it remains unclear whether and to what extent the identified microRNAs are relevant to the clinical condition of AKI in human patients.

In the present study, we have verified the induction of microRNA-668 (miR-668) in ischemic AKI using human patient samples as well as animal and cell models. miR-668 is evolutionarily conserved, but its function and regulation are largely unknown, except a recent report suggesting its involvement in the radioresistance of human breast cancer cells (41). In the current study, we show that miR-668 is induced via HIF-1 during ischemic AKI. Upon induction, miR- 668 represses the mitochondrial fission protein MTP18 to prevent mitochondrial fragmentation and protect renal tubular cells from apoptosis. Together, these results reveal the HIF-1/miR-668/MTP18 pathway as a novel protective mechanism and a potential therapeutic target in ischemic AKI.

\section{Results}

miR-668 is induced in ischemic AKI. Our previous work has identified a list of microRNAs with significant changes of expression during ischemic AKI in mice (24). miR-668 is one of the highly induced microRNAs after 30 minutes of bilateral renal ischemia with 48 hours of reperfusion (I30/48h). To determine its clinical relevance, we examined miR-668 in human patients with or without AKI. AKI in these patients was indicated by elevated levels of serum creatinine and blood urea nitrogen (BUN) (Supplemental Figure 1A; supplemental material available online with this article; https://doi.org/10.1172/JCI121859DS1). As shown in Figure $1 \mathrm{~A}$, the kidney biopsies from AKI patients had significantly higher miR-668 than those of non-AKI patients (Figure 1A). These 2 groups showed a similar tendency of miR-668 in their urine and blood samples (Supplemental Figure 1, B and C). In addition, kidney miR-668 expression showed a good correlation with the levels of serum creatinine and BUN in these patients (Figure 1B $[r=0.5285, P=0.0454$, Spearman's correlation test $]$ and Figure 1C $[r=0.5201, P=0.0491$, Spearman's correlation test $])$. To specifically examine patients with renal ischemia/reperfusion, we further analyzed miR-668 in urine and serum samples from the patients of cardiac surgery with cardiopulmonary bypass and aortic cross-clamping (Supplemental Figure 2). These patients were categorized into AKI and non-AKI groups according to their post- surgery serum creatinine levels (Supplemental Figure 2A). Compared with their presurgery levels, both AKI and non-AKI patients showed miR-668 increases in serum after cardiac surgery, and the levels of serum miR-668 increase were comparable in these patients (Figure 1D). In urine, the patients with postsurgery AKI showed a significant miR-668 increase, whereas the non-AKI patients did not. Compared with non-AKI patients, the patients with postsurgery AKI in general had more miR-668 in urine (Figure 1E). Because of ethical issues, it was not possible to collect kidney tissues to determine miR-668 in these patients. However, higher urine micro-668 in the patients with AKI suggests miR-668 induction in human kidneys during cardiopulmonary bypassassociated renal ischemia/reperfusion.

We further verified miR-668 induction in ischemic AKI in mice by TaqMan-based quantitative real-time PCR (qPCR) (Figure $1 F)$. miR-668 was induced significantly at I30/12h and marginally at $\mathrm{I} 30 / 48 \mathrm{~h}$. Our in situ hybridization analysis localized miR-668 induction in ischemic AKI in renal tubules in cortex and outer medulla with relatively intact tubular structure (Figure $1 \mathrm{H}$ ). Most of the tubules had brush border indicating proximal tubules. We also detected miR-668 induction during 3-6 hours of hypoxia $\left(1 \% \mathrm{O}_{2}\right)$ in cultured rat proximal tubular cells (RPTCs) (Figure $1 \mathrm{G}$ ).

HIF-1 mediates miR-668 induction in ischemic AKI. To understand the mechanism of miR-668 induction in ischemic AKI, we examined its transcriptional regulation. Analysis by the JASPAR database revealed the binding sites of multiple transcription factors at the $5^{\prime}$ upstream sequence of the miR- 668 gene, which included HIF-1, a "master regulator" of gene expression during ischemia/hypoxia $(42,43)$. HIF-1 induction occurs in renal tubule cells during and following ischemic AKI $(36,44)$. To determine the role of HIF-1 in miR-668 induction, we initially compared WT (HIF-1 ${ }^{+/+}$) and HIF-1-knockout (HIF-1-/-) mouse embryonic fibroblasts (MEFs). As expected, HIF-1 $\alpha$ was induced by hypoxia in $\mathrm{HIF}-1^{+/+}$cells but not in $\mathrm{HIF}-1^{-/-}$cells (Figure $2 \mathrm{~A}$ ). Importantly, miR-668 was induced by hypoxia in $\mathrm{HIF}^{-1^{+/+}}$MEF cells, but not in HIF-1/- cells (Figure 2B). We further compared miR-668 induction by ischemic AKI in WT $\left(\mathrm{PT}-\mathrm{HIF}-1^{+/+}\right)$and proximal tubule-specific HIF-1 $\alpha$-knockout (PT-HIF-1/-) mice. The blockade of HIF-1 induction in PT-HIF-1/-- kidneys during ischemic AKI has been confirmed in our previous study (36). I30/48h induced an over 2-fold increase of miR-668 in WT mouse kidneys, which was markedly suppressed in PT-HIF-1 ${ }^{-/-}$mice (Figure 2C). HIF-1 induction along with miR-668 upregulation was also identified in another kidney injury model of unilateral ureteral obstruction (UUO). At 4 days after UUO, there was obvious induction of HIF-1 $\alpha$ in kidneys, accompanied by significant upregulation of miR-668 (Supplemental Figure 3).

Analysis with the JASPAR database predicted multiple hypoxia response elements (HREs) or HIF-1 binding sites in the promoter region of the miR-668 gene. We therefore examined HIF-1 binding to 2 sites with the highest score by chromatin immunoprecipitation (ChIP) assay (Figure 2D and Table 1). HIF-1 ${ }^{+/+}$and HIF-1/cells were incubated under hypoxia or normoxia for 6 hours. The DNA fragments pulled down by specific HIF-1 $\alpha$ antibody were examined by qPCR. Upon hypoxia, significantly more site 1 DNA fragments were pulled down by HIF- $1 \alpha$ antibodies in HIF-1 ${ }^{+/+}$cells $(4.45 \pm 3.68$-fold increase, $P=0.0348)$, but not in $\mathrm{HIF}-1^{-/-}$cells 
A

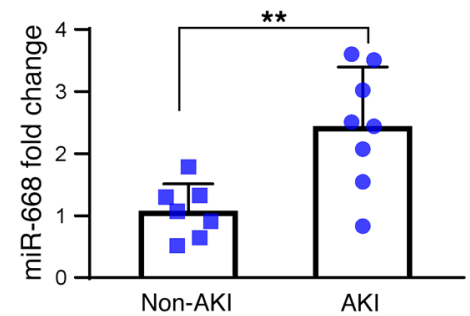

D

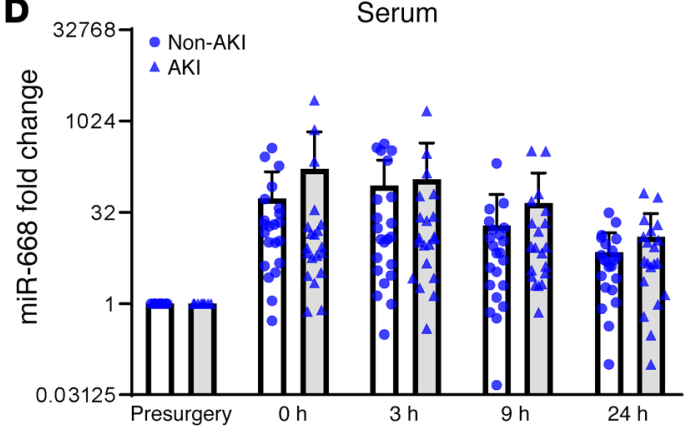

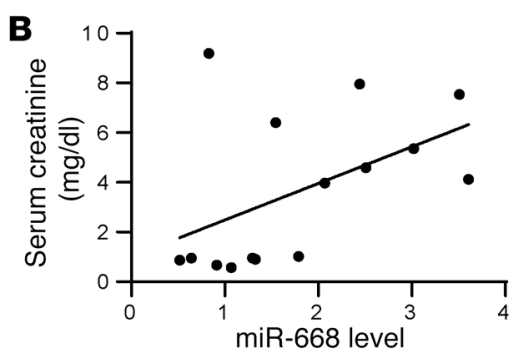

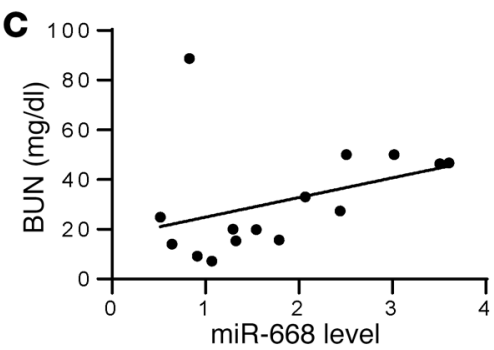

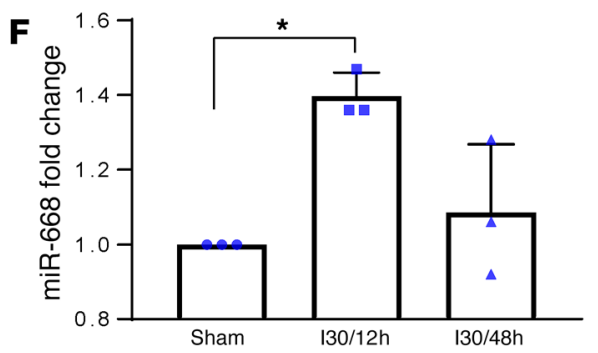

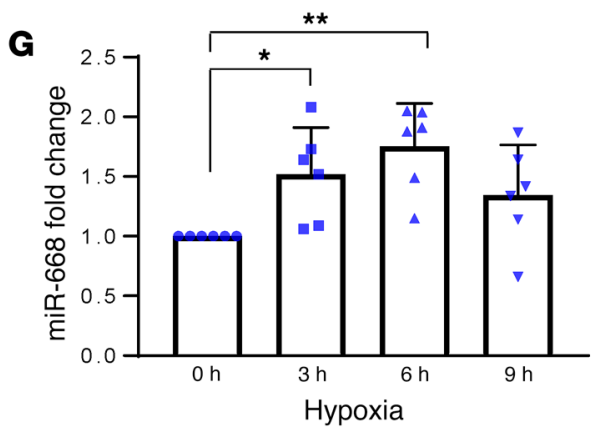

H

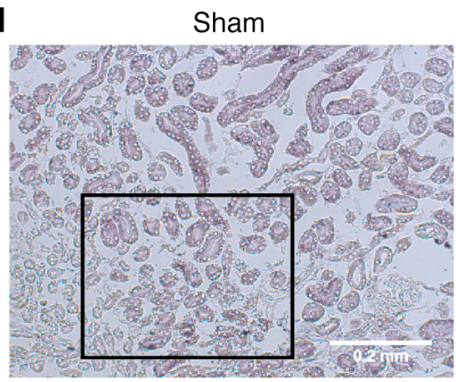

E
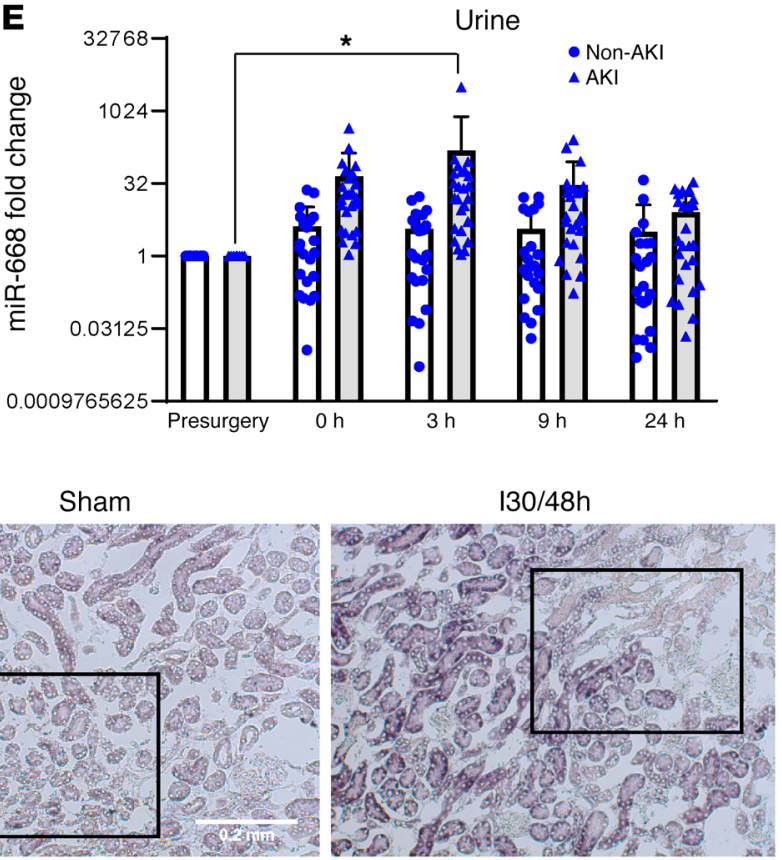
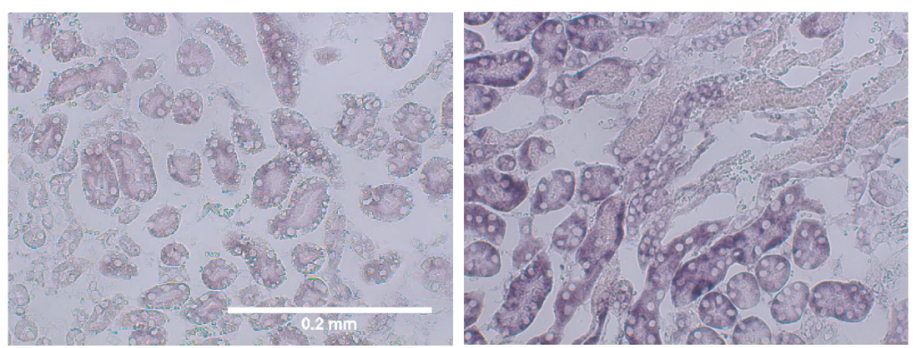

Figure 1. miR-668 is induced in ischemic AKI. (A) qPCR analysis of kidney biopsies showing 2.5-fold higher miR-668 in AKI patients versus non-AKI patients ( $n=8$ for AKI group, $n=7$ for non-AKI group; ${ }^{* *} P=0.0046$, 2-tailed Student's $t$ test). (B) Correlation between kidney miR-668 and serum creatinine in patients $(r=0.5285, P=0.0454$, Spearman's correlation test). (C) Correlation between kidney miR-668 and blood urea nitrogen (BUN) level ( $r=$ $0.5201, P=0.0491$, Spearman's correlation test). (D) qPCR analysis of miR-668 in serum samples collected from patients before or at different time points after cardiac surgery ( $n=20$ for AKI, $n=22$ for non-AKI). (E) miR-668 in urine samples collected from patients before or at different time points after cardiac surgery ( $n=25$ for AKI, $n=22$ for non-AKI; ${ }^{*} P=0.0089,2$-way ANOVA with Fisher's LSD). (F) qPCR analysis of miR-668 in mouse kidneys with 30 minutes of bilateral renal ischemia and 12 hours $(I 30 / 12 \mathrm{~h})$ or 48 hours $(I 30 / 48 \mathrm{~h})$ of reperfusion, or sham operation $\left(n=3 ;{ }^{*} P=0.0447,1\right.$-way $A N O V A$ with Dunn's multiple-comparisons test). (G) qPCR analysis of miR-668 in RPTCs after $0-9$ hours of hypoxia $(1 \% 02)$ treatment $\left(n=6\right.$; ${ }^{*} P=0.0269,{ }^{*} P=0.0016$, 1-way ANOVA with Dunn's multiple-comparisons test). (H) In situ hybridization showing miR-668 induction in the cells of relatively intact renal tubules during ischemic AKI in mice $(n=2)$. Bottom panels are enlarged images of the boxed areas in the top panels. Scale bar: 0.2 mm.

(Figure 2D). In contrast, the pull-down of site 2 DNA fragments only increased marginally after hypoxia treatment $(2.08 \pm 1.65-$ fold increase, $P=0.085, t=2.141$, degrees of freedom $[\mathrm{DF}]=5$ ). The results indicate that HIF-1 may mediate miR-668 induction in renal ischemia/hypoxia by directly binding to the gene promoter for transcriptional activation.
miR-668 is antiapoptotic in kidney proximal tubular cells. Transient transfection of scrambled-sequence locked nucleic acid (LNA) in RPTCs induced some apoptosis due to the toxicity of the transfection procedure with Lipofectamine 2000 (Figure 3, A-C). Notably, transfection of anti-miR-668 in LNA induced significantly more apoptosis as indicated by cell morphology and caspase 
A
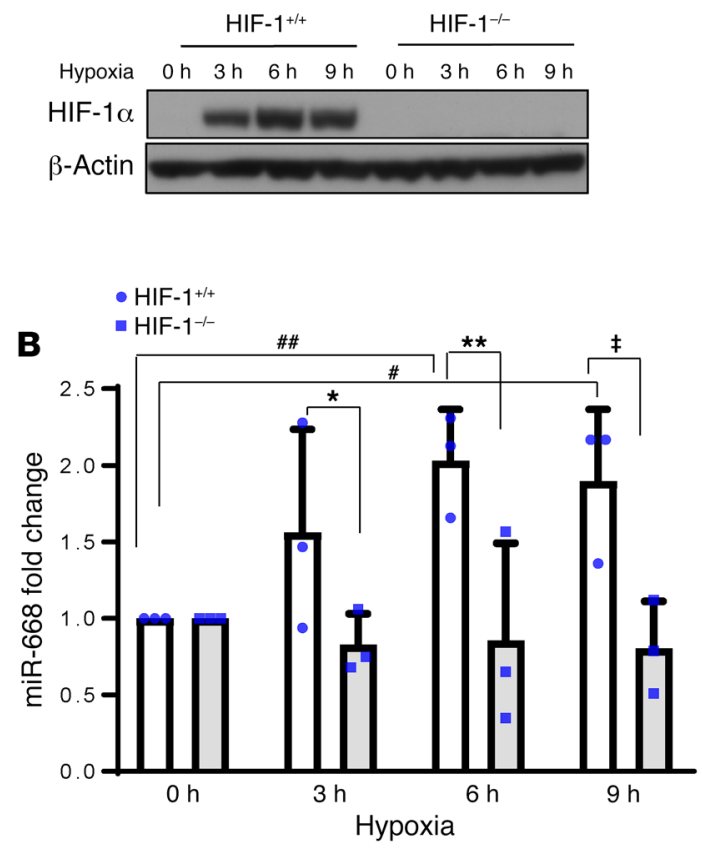

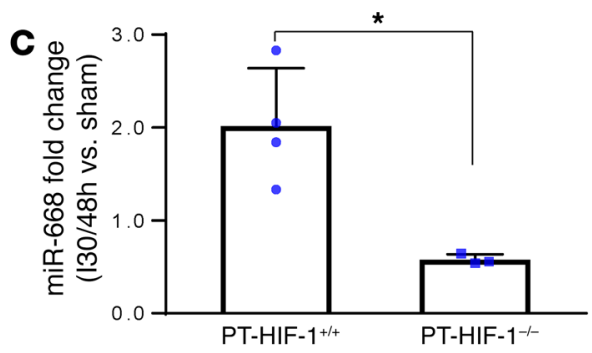

D

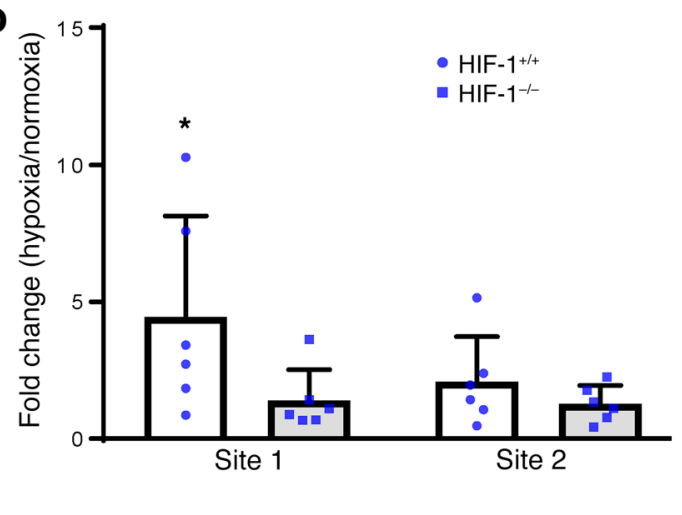

Figure 2. HIF-1 mediates miR-668 induction in ischemic AKI. (A) Immunoblots showing HIF-1 $\alpha$ induction by $0-9$ hours of $1 \%$ O hypoxia in HIF- $1 \alpha^{+/+}$MEF cells, but not in HIF-1 $\alpha^{-/-}$cells. Experiments were repeated twice. (B) qPCR analysis showing the induction of miR-668 by hypoxia in HIF-1 $\alpha^{+/+}$MEF cells, but not in HIF-1 $\alpha{ }^{-/-}$cells $\left(n=3 ;{ }^{\#} P=0.0118,{ }^{\#} P=0.0052,{ }^{*} P=0.0423,{ }^{*} P=0.0027,{ }^{\ddagger} P=0.0046,2\right.$-way ANOVA with Fisher's LSD). (C) qPCR analysis showing a 2-fold increase of miR-668 during $130 / 48 \mathrm{~h}$ ischemic AKI in WT (PT-HIF-1 $\alpha^{+/+}$) mice $(n=4)$ that was suppressed to 0.5 -fold in proximal tubule-specific HIF-1 $\alpha$-knockout (PT-HIF-1 $\left.\alpha^{-l-}\right)$ mice $\left(n=3 ;{ }^{*} P=0.0185\right.$, 2-tailed Student's $t$ test). (D) ChIP assay showing hypoxia-induced increase of HIF-1 binding to the predicted site 1 (and not site 2 ) of the miR-668 gene promoter in HIF-1 $\alpha^{+/+}$cells, but not in HIF-1 $\alpha^{-/-}$cells $\left(n=6\right.$; ${ }^{*} P=0.035$, 1 -tailed Student's $t$ test [hypoxia vs. normoxia in HIF-1+/+ cells]).

activation (Figure $3, \mathrm{~A}^{-} \mathrm{C}$ ), indicating a cytoprotective role of miR668. We further examined the effect of miR-668 mimic (Figure 3, D-F). In control condition, miR-668 mimic did not have a noticeable effect on cell death or survival. However, the mimic attenuated apoptosis and caspase activation induced by ATP depletion in RPTCs (Figure 3, D-F). We also examined how miR-668 contributes to HIF-1-dependent cellular protection (Supplemental Figure 4). HIF-1 $\alpha$ induction by hypoxia in RPTCs was suppressed by HIF-1 $\alpha$ shRNA stable transfection (Supplemental Figure 4A). After 48 hours of hypoxia, HIF-1 $\alpha$-knockdown cells showed obviously more apoptosis, which was totally reversed by overexpression of miR-668 (Supplemental Figure 4, B-D), supporting a pivotal role of miR-668 in HIF-1-mediated cellular protection.

Inhibition of miR-668 exacerbates ischemic AKI. To determine the role of miR-668 in vivo, we first tested the effect of anti-miR668 LNA on ischemic AKI in mice. Anti-miR-668 LNAs or scrambled-sequence oligonucleotides were administered to mice at 20 $\mathrm{mg} / \mathrm{kg} 2$ days before renal ischemia (Figure 4A). As expected, ischemic AKI was associated with miR-668 induction, which was abrogated by anti-miR-668 LNAs (Figure 4B). Anti-miR-668 LNA did not affect renal function or histology in sham-operated control mice, but it increased AKI following renal ischemia/reperfusion (Figure 4, C-F). Compared with scrambled oligonucleotide treatment, anti-miR-668 induced higher BUN (244.48 \pm 50.67 vs. $328.23 \pm 63.93 \mathrm{mg} / \mathrm{dl})$ and serum creatinine $(2.06 \pm 0.40 \mathrm{vs} .2 .75 \pm$ $0.29 \mathrm{mg} / \mathrm{dl})$. Anti-miR-668 also increased BUN and serum creat- inine in the moderate AKI model of 25 minutes of renal ischemia/ reperfusion (Supplemental Figure 5). Interestingly, anti-miR668 did not have obvious effects on histological damage in renal tubules shown by H\&E staining (Figure $4 \mathrm{~A}$ and Supplemental Figure 6A). Nonetheless, anti-miR-668 increased renal apoptosis in ischemic AKI as shown by TUNEL staining (Figure $4, \mathrm{~F}$ and $\mathrm{G}$ ) and active caspase- 3 immunoblotting (Figure $4 \mathrm{H}$ ).

miR-668 mimic protects kidneys from ischemic AKI. To further establish the role of miR- 668 and determine its therapeutic potential, we tested the effects of miR-668 mimic in ischemic AKI in mice (Figure $5 \mathrm{~A}$ ). In vivo delivery of miR-668 mimic resulted in a sustained increase of miR-668 in kidney tissues, including both renal cortex and medulla (Figure 5B and Supplemental Figure 7). miR-668 mimic did not affect renal function and histology in sham-operated mice, but it ameliorated ischemic AKI (Figure 5, C-F). Functionally, miR-668 mimic reduced BUN (301.29 \pm 17.46

Table 1. Information of 2 HIF-1 binding sites at miR-668 gene
promoter predicted by JASPAR database

$\begin{array}{lcccc}\text { miR-668 } & \text { JASPAR score } & \text { Start } & \text { End } & \text { Sequence } \\ \text { Site } 1 & 9.542 & -566 & -559 & \text { GGGCGTGC } \\ \text { Site 2 } & 8.295 & -58 & -51 & \text { GGCCGTCC }\end{array}$


A
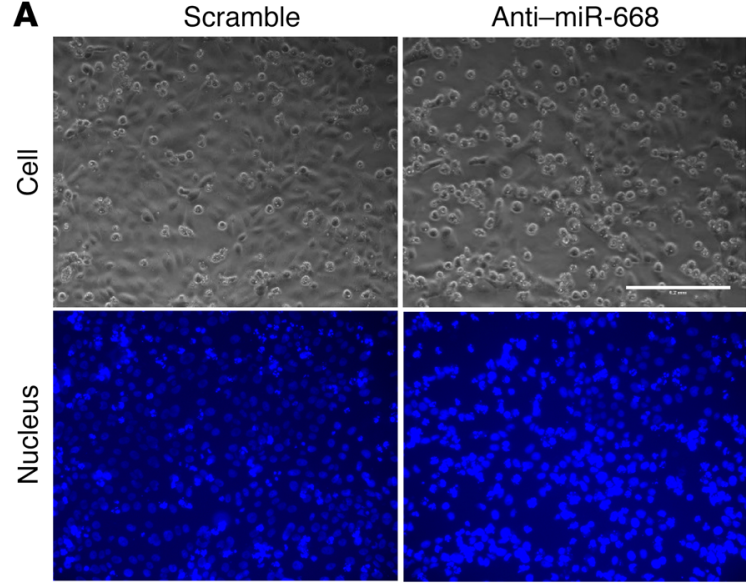

D
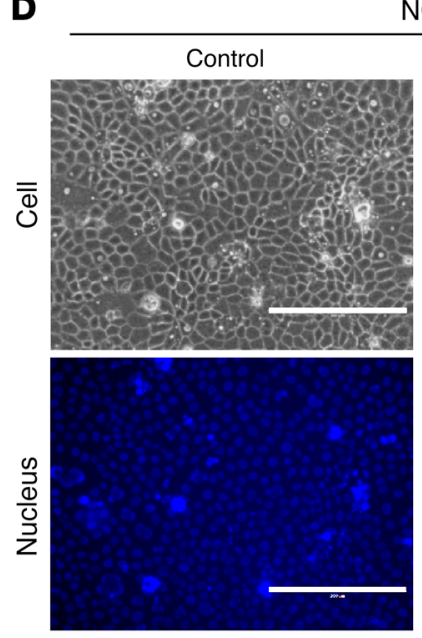

$\mathrm{NC}$
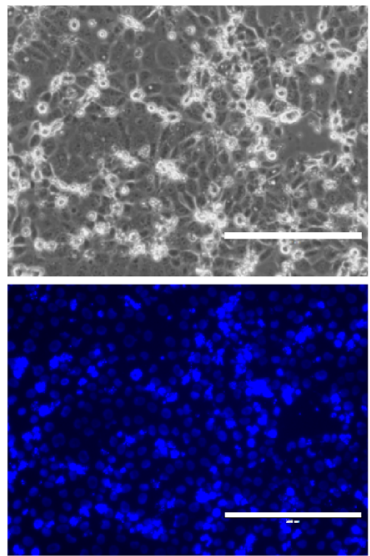

E

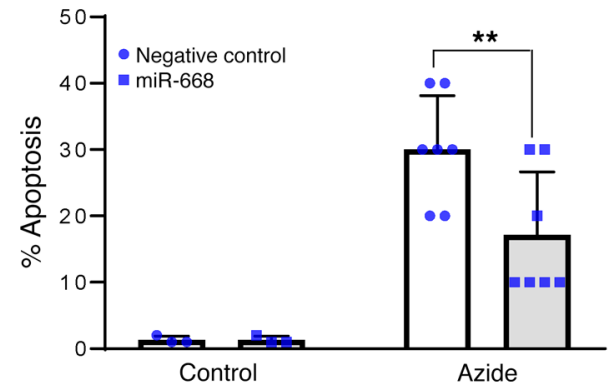

B
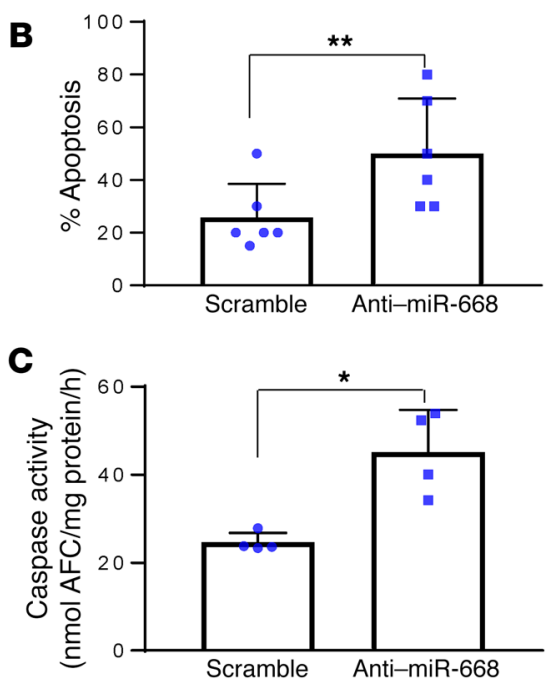

\begin{tabular}{cc} 
miR-668 & \\
\hline Control & Azide
\end{tabular}
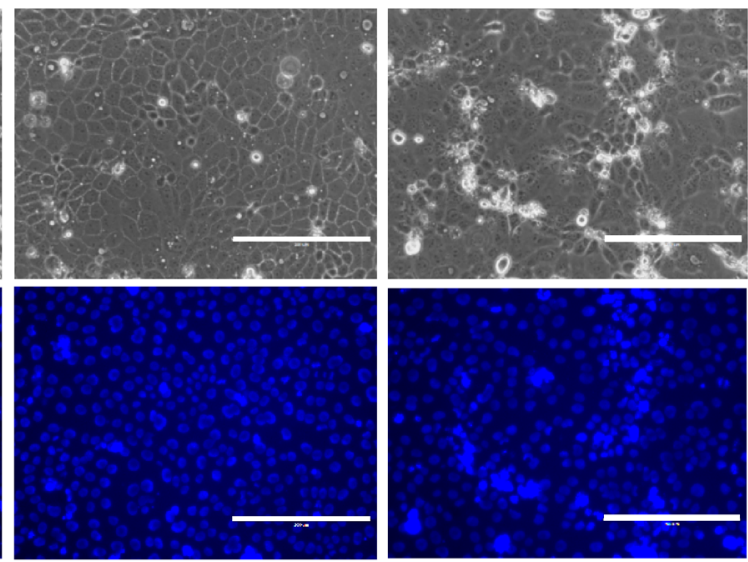

$\mathbf{F}$

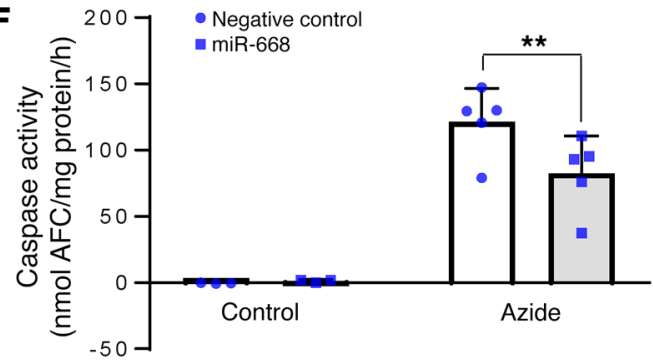

Figure 3. miR-668 is antiapoptotic in kidney proximal tubular cells. (A) Representative cell and nuclear images of RPTCs showing the induction of apoptosis by 200 nM anti-miR-668, but not by scrambled-sequence LNAs. Scale bar: 0.2 mm. (B) Percentage of apoptosis in anti-miR-668-treated or scrambled-sequence LNA-treated cells assessed by counting of the cells with typical apoptotic morphologies ( $n=6$; ${ }^{*} P=0.00316$, 2-tailed Student's $t$ test). (C) Caspase activity showing the induction of caspase activation by anti-miR-668 ( $n=4$; ${ }^{*} P=0.030$, 2-tailed Student's $t$ test). (D) Representative cell and nuclear images of RPTCs showing the antiapoptotic effect of $200 \mathrm{nM}$ miR-668 mimics (miR-668), but not the negative control (NC) oligonucleotides. The cells were treated with $10 \mathrm{mM}$ azide for 3 hours to induce ATP depletion and then recovered for 2 hours in full culture medium to develop apoptosis (Azide). Scale bars: $0.2 \mathrm{~mm}$. (E) Percentage of apoptosis assessed by counting of the cells with typical apoptotic morphologies ( $n=3$ for control groups, $n=7$ for azide groups; ${ }^{*} P=0.0004,2$-tailed Student's $t$ test). (F) Caspase activity verifying the antiapoptotic effect of miR-668 ( $n=3$ for control groups, $n=5$ for azide groups; ${ }^{*} P=0.0011,2$-tailed Student's $t$ test).

vs. $224.45 \pm 62.93 \mathrm{mg} / \mathrm{dl})$ and serum creatinine $(2.33 \pm 0.58$ vs. $1.49 \pm 0.54 \mathrm{mg} / \mathrm{dl}$ ) during renal ischemia/reperfusion (Figure 5, $\mathrm{C}$ and $\mathrm{D})$. The mimic did not significantly affect the histological damage in renal tubules (Figure $5 \mathrm{E}$ and Supplemental Figure
6B); however, it attenuated renal apoptosis as shown by TUNEL assay and active caspase- 3 (Figure $5, \mathrm{~F}-\mathrm{H}$ ). Together, these results suggest that miR-668 is induced in ischemic AKI to protect renal tubular cells from apoptosis. 
A

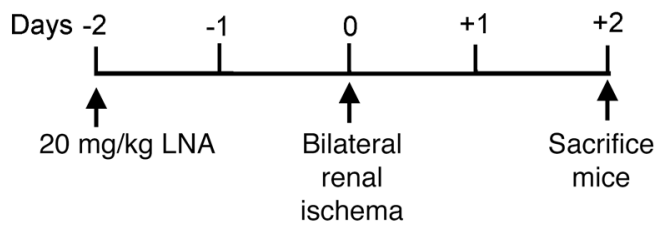

C

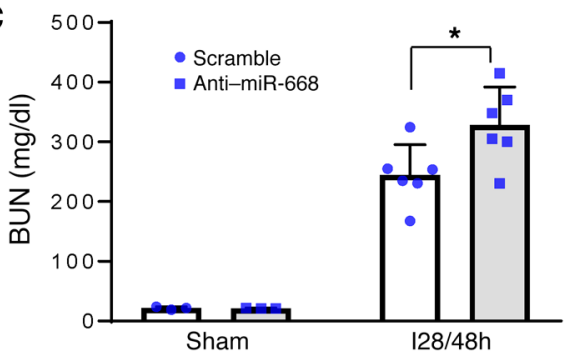

E

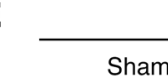

Scramble

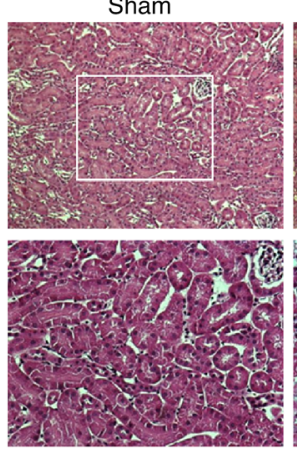

$\mathbf{F}$
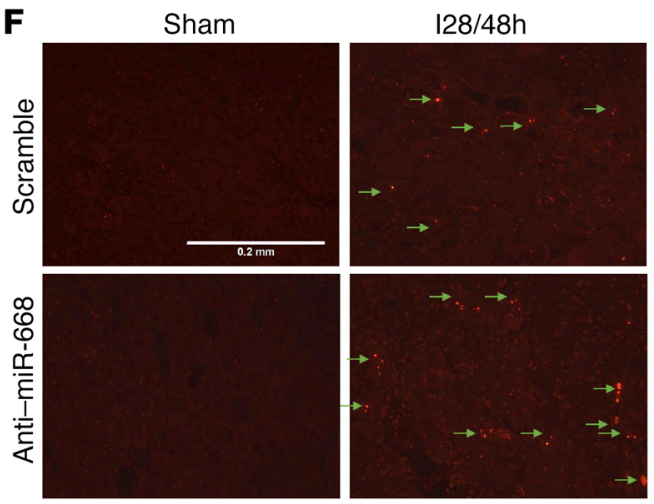

B

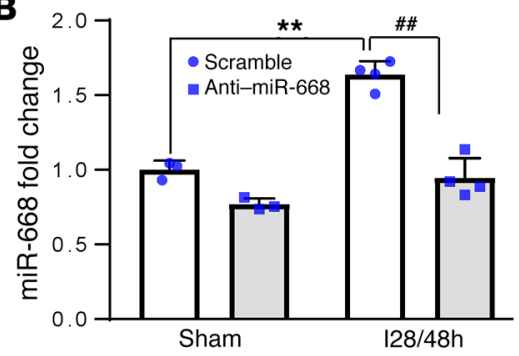

D
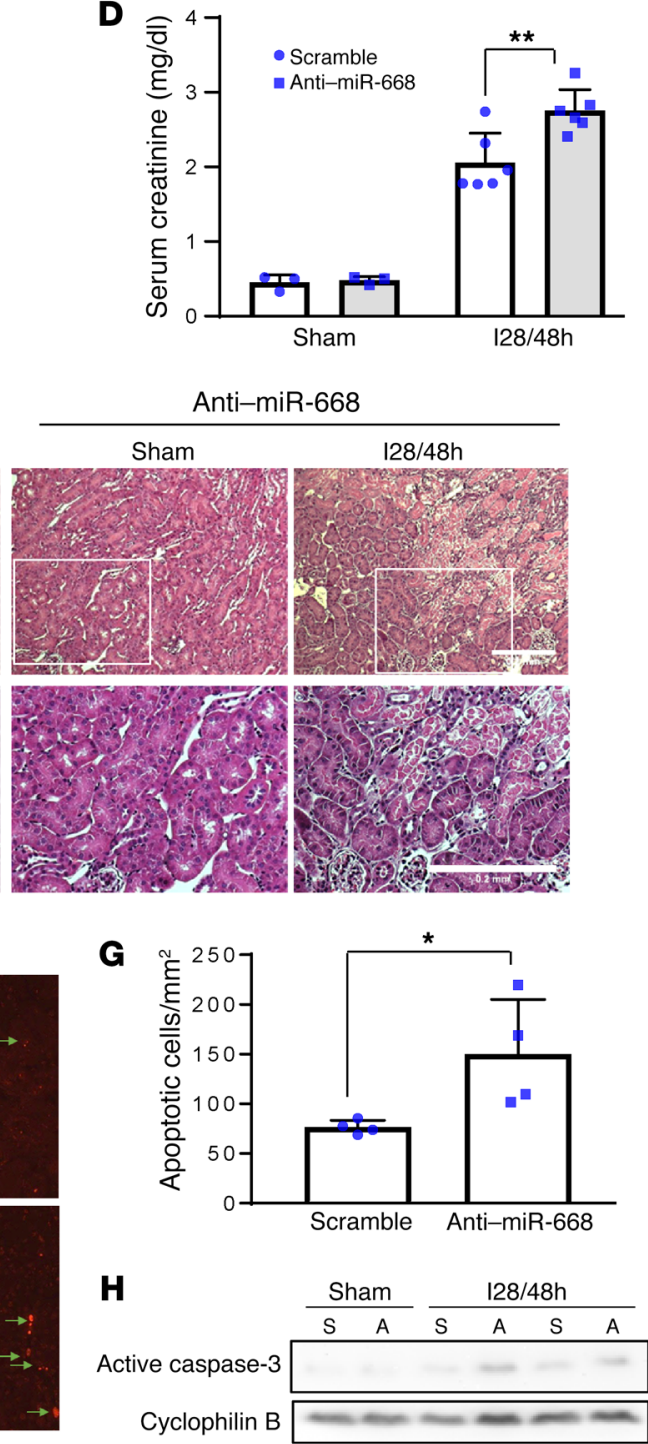

Figure 4. Inhibition of miR-668 exacerbates ischemic AKI. (A) Schematic diagram depicting the animal treatment procedure. Anti-miR-668 or scrambled-sequence LNAs were delivered to C57BL/6] male mice through tail vein injection 2 days before ischemic surgery. The mice were subjected to 28 minutes of bilateral renal ischemia followed by 48 hours of reperfusion (128/48h), or sham operation. (B) qPCR analysis of miR-668 in kidneys verifying the inhibitory effect of anti-miR-668 ( $n=3$ for sham groups and $n=4$ for $128 / 48$ h groups; ${ }^{* *} P<$ $0.0001, " \# P<0.0001$, 2-way ANOVA with Fisher's LSD). (C) BUN measurement showing ischemic AKI exacerbated by anti-miR-668 ( $n=3$ for sham groups, $n=6$ for $128 / 48$ h groups; ${ }^{*} P=0.0318$, 2-tailed Student's $t$ test). (D) Serum creatinine measurement showing ischemic AKI exacerbated by anti-miR-668 ( $n=3$ for sham groups, $n=6$ for $128 / 48 \mathrm{~h}$ groups; ${ }^{*} P=0.007,2$-tailed Student's $t$ test). (E) Representative images of renal histology by H\&E staining. Bottom panels are enlarged images of the boxed areas in the top panels. Scale bars: 0.2 $\mathrm{mm}$. (F) Representative images of TUNEL staining. The arrows indicate TUNELpositive nuclei. Scale bar: $0.2 \mathrm{~mm}$. (G) Counting of TUNEL-positive cells to show that anti-miR-668 increased apoptosis in ischemic AKI $\left(n=4 ;{ }^{*} P=0.0386\right.$, 2-tailed Student's $t$ test). (H) Immunoblots showing the induction of active caspase-3 by anti-miR-668 in ischemic AKI. S, scramble; A, anti-miR-668. Cyclophilin B was used as loading control.
miR-668 protects against mitochondrial fragmentation during renal cell stress. How does miR-668 protect renal tubular cells in ischemic AKI? In this regard, we had an interesting observation that suggested a role of miR-668 in preserving mitochondrial dynamics during cell stress. Disruption of mitochondrial dynamics is known to induce mitochondrial fragmentation, which contributes to mitochondrial damage and consequent activation of apoptosis in disease conditions of the kidney, heart, and brain $(4,7$, 10-12). In our experiments, anti-miR-668 significantly increased the number of RPTCs with fragmented mitochondria (from $37.1 \%$ $\pm 12.0 \%$ to $61.1 \% \pm 12.3 \%$ ) (Figure $6, \mathrm{~A}$ and B). Similarly, anti-miR-
668 increased mitochondrial fragmentation in HEK293 cells (Figure $6 \mathrm{C}$ ). On the contrary, miR-668 mimic could suppress mitochondrial fragmentation during hypoxia stress in HEK293 cells (Figure 6D), further indicating a role of miR-668 in maintaining mitochondrial dynamics and morphology during cell stress.

Mitochondrial dynamics is governed by mitochondrial fission and fusion proteins $(14,15,45)$. To elucidate the regulation of miR668 , we initially examined its effects on several well-documented mitochondrial fission and fusion proteins. As shown in Figure 6E, miR-668 did not affect the expression and phosphorylation of Drp1, a mitochondrial fission protein. It did not affect the expression of 

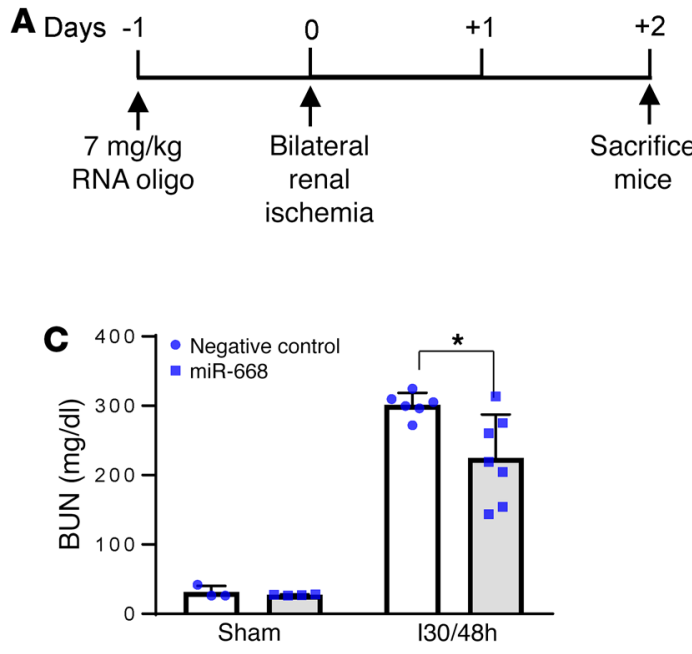

E

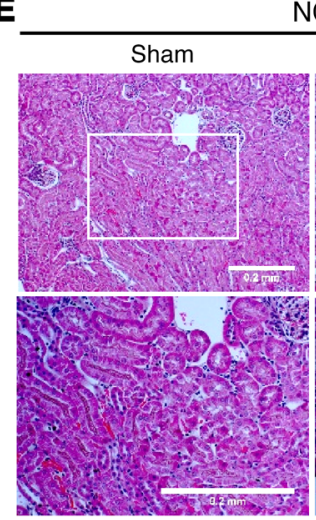

NC

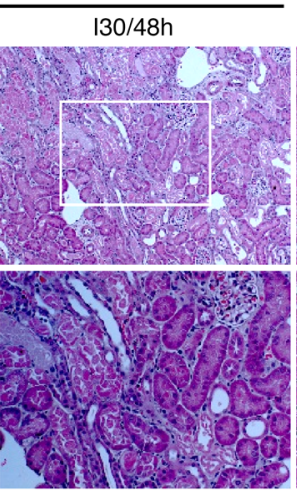

$\mathbf{F}$
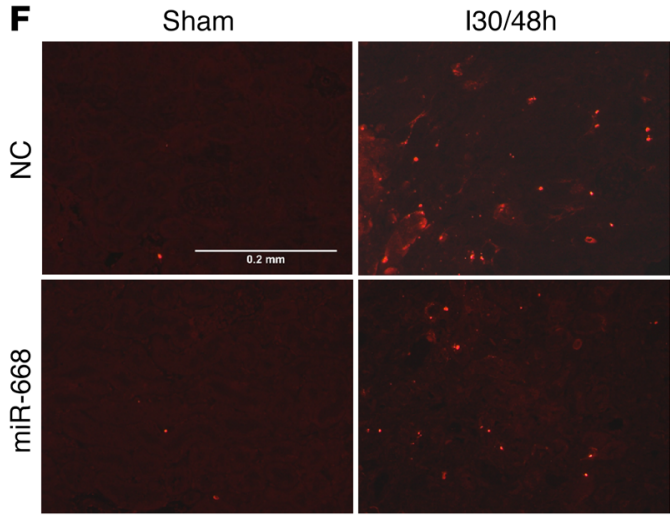

B

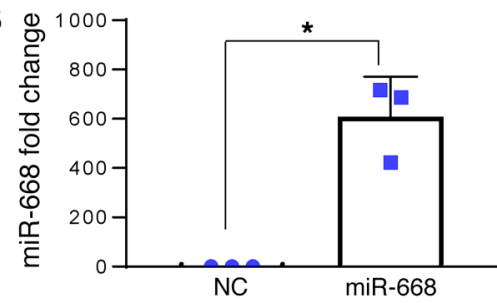

D
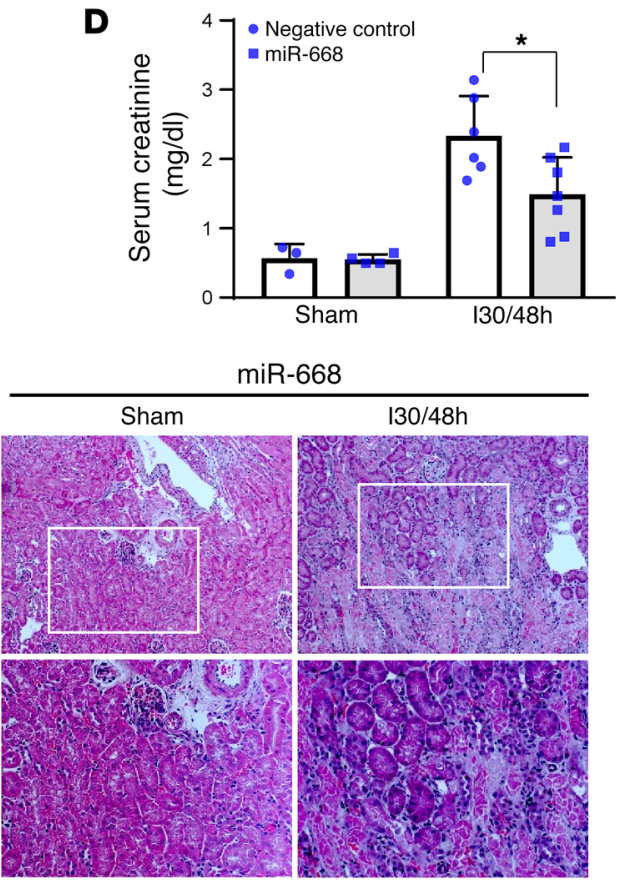

G

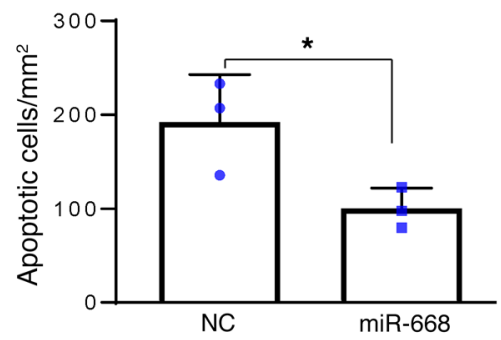

H

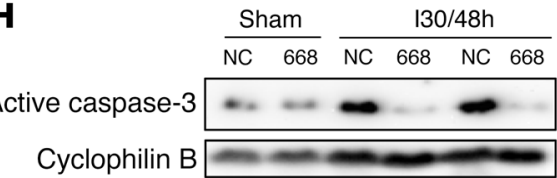

Figure 5. miR-668 mimic protects kidneys from ischemic AKI. (A) Schematic diagram depicting the animal treatment procedure. miR668 or negative control (NC) RNA oligonucleotides were delivered to [57BL/6] male mice through tail vein injection 1 day before ischemic surgery. The mice were subjected to 30 minutes of bilateral renal ischemia with 48 hours of reperfusion (130/48h), or sham operation. (B) qPCR of miR-668 to verify successful RNA oligonucleotide delivery to mouse kidneys ( $n=3 ;{ }^{*} P$ $=0.023,2$-tailed Student's $t$ test). (C) BUN measurement showing the protective effect of miR-668 $(n=3$ for sham group negative control, $n=4$ for sham group with miR-668, $n=6$ for $130 / 48$ h negative control, $n=7$ for $130 / 48$ h with miR-668; ${ }^{*} P=0.045$ for $130 / 48$ h, 2-tailed Student's $t$ test). (D) Serum creatinine levels showing the protective effect of miR-668 ( $n=3$ for sham group negative control, $n=4$ for sham group with miR-668, $n=6$ for 130/48h with negative control, $n=7$ for $130 / 48$ h with miR-668; ${ }^{*} P=0.021,2$-tailed Student's $t$ test). (E) Representative images of renal histology by H\&E staining. Bottom panels are enlarged images of the boxed areas in the top panels. Scale bars: $0.2 \mathrm{~mm}$. (F) Representative images of TUNEL staining. Scale bar: $0.2 \mathrm{~mm}$. (G) Counting of TUNEL-positive cells to show the antiapoptotic effect of miR-668 in ischemic AKI ( $n=3 ;{ }^{*} P=0.0446$, 2-tailed Student's $t$ test). (H) Immunoblots showing the inhibition of caspase-3 cleavage in ischemic AKI kidney with miR-668 overexpression. NC, negative control; 668, miR-668 mimics. Cyclophilin B was used as loading control. mitofusin 1, mitofusin 2, or OPA1, three mitochondrial fusion proteins, either. Moreover, miR-668 did not affect Bak, a proapoptotic Bcl-2 family protein that mediates mitochondrial fragmentation during cell stress by interacting with mitofusins $(19,46)$.

miR-668 targets MTP18 in ischemic AKI and renal tubular cells. With these negative results, we decided to systemically analyze the gene targets of miR-668 by microRNA-induced silencing complex (miRISC) immunoprecipitation followed by RNA deep sequencing. To this end, HEK293 cells were cotransfected with FLAG-tagged argonaute-2 (Ago-2, a key protein in miRISC) and miR-668 mimic or its negative control RNA oligonucleotides to collect cell lysate for immunoprecipitation with anti-FLAG anti- body. The mRNAs associated with miRISC in the presence or absence of miR-668 were analyzed by deep RNA sequencing (Figure 7A). As shown in Figure 7A, miR-668 transfection resulted in significant ( $>2$-fold) accumulation of 680 RNA species in miRISC, of which 407 were transcribed from protein-coding genes while 273 were from pseudogenes, unnamed genes, or noncoding sequences. The deep sequencing also showed the accumulation of a few other microRNAs in miRISC. To further identify the targets of miR-668, we analyzed the $3^{\prime}$-UTRs of the 407 protein-coding genes with the miRanda and Segal Lab of Computational Biology microRNA target prediction databases, and found that 124 of them contained at least one miR-668-targeting seed sequence (Supple- 
A
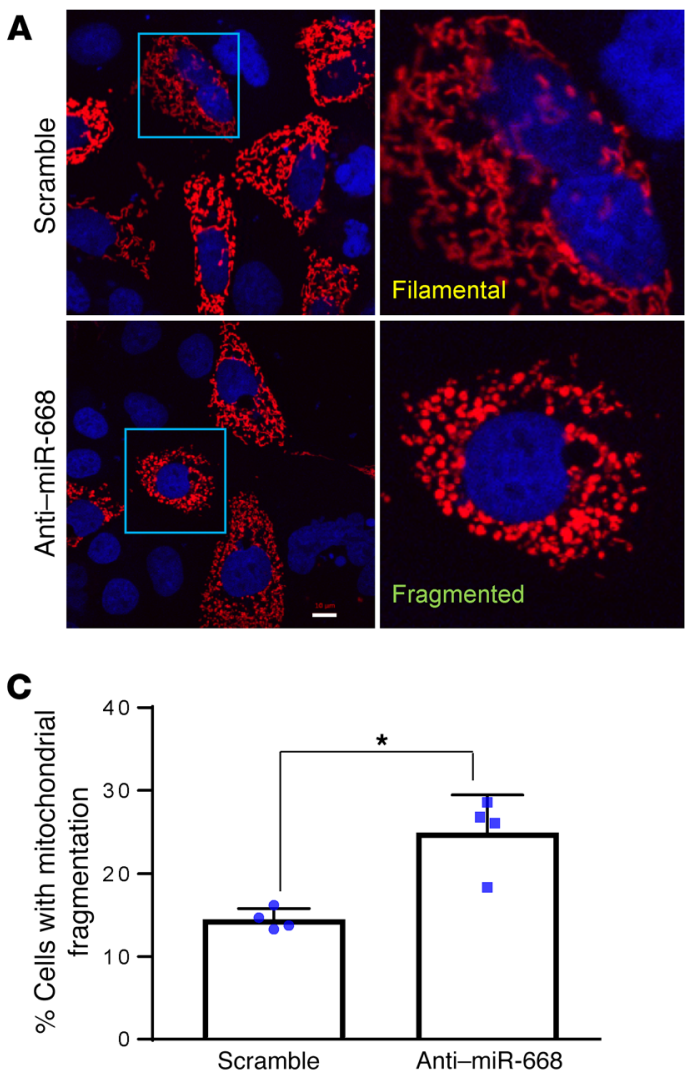

B
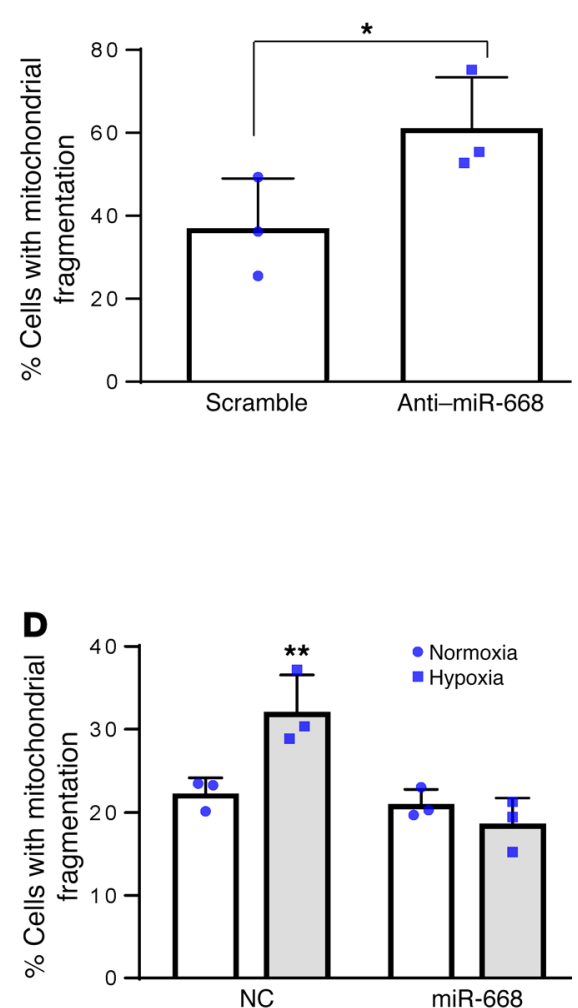

Figure 6. miR-668 protects against mitochondrial fragmentation during renal cell stress. (A) Representative morphology of mitochondria (labeled by Mito-Red in red) in RPTCs transfected with anti-miR-668 or scrambled LNAs. Cell nuclei were labeled by Hoechst in blue. Right panels are enlarged images of the boxed areas in the left panels. Scale bar: $10 \mu \mathrm{m}$. (B) Percentage of RPTCs with mitochondrial fragmentation showing the enhancing effect of anti-miR-668 ( $n=3$; ${ }^{*} P=0.0107$, 2-tailed Student's $t$ test). (C) Percentage of mitochondrial fragmentation in HEK293 cells showing the enhancing effect of anti-miR-668 ( $n=4 ;{ }^{*} P=0.0152$, 2-tailed Student's $t$ test). (D) Percentage of mitochondrial fragmentation in HEK293 cells showing the inhibitory effect of miR-668 mimic (miR-668) in comparison with negative control (NC) RNA oligonucleotides. The cells were treated with or without $1 \% \mathrm{O}_{2}$ hypoxia for 6 hours $(n=3$; ${ }^{*} P=0.0027$, 1-way ANOVA). (E) Immunoblots of mitochondrial dynamic regulation proteins from RPTCs with negative control (NC) or miR-668 mimic transfection. Cyclophilin B or $\beta$-actin was used as loading control.

$\mathbf{E}$

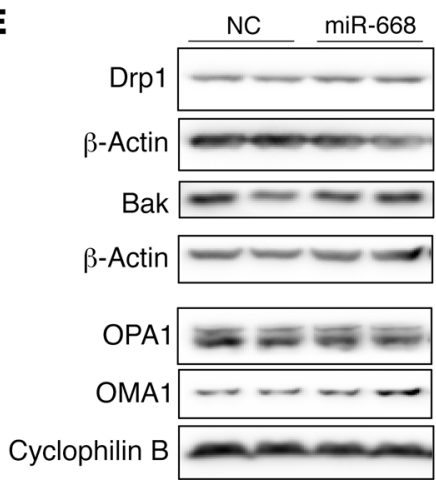

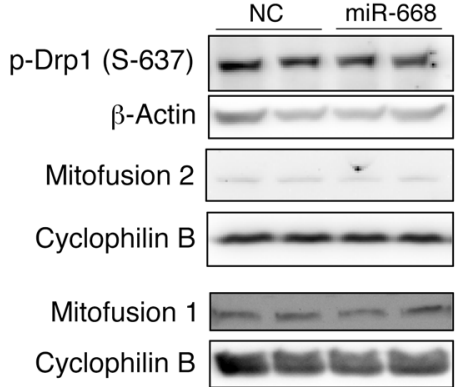

mental Table 1). Remarkably, of these 124 genes, MTFP1 (encoding MTP18; RISC accumulation fold $2.31 \pm 0.51, n=3$ ) was the only one that has been implicated in the regulation of mitochondrial dynamics by promoting mitochondrial fission (16). The Segal Lab database further predicted multiple miR-668 targeting sites in the 3 '-UTR of MTP18 mRNA in human, mouse, and rat (Supplemental Table 2). Experimentally, miR-668 mimic suppressed MTP18 expression in HEK293 cells (Figure 7B), whereas anti-miR-668 increased MTP18 (Figure 7C). In vivo, MTP18 decreased in both ischemic AKI and UUO injury when miR-668 was induced, suggesting a connection between miR-668 and MTP18 (Figure 7, D and E, and Supplemental Figure 3A). Anti-miR-668 marginally increased renal MTP18 expression in sham-operated control mice and showed no effect on MTP18 in ischemic AKI (Figure 7D). However, miR-668 mimic significantly reduced renal MTP18 expression in both sham-operated mice and ischemic AKI mice (Figure 7E), further validating MTP18 as a target of miR-668. We further analyzed 2 predicted miR-668 targeting sites in the human MTP18 gene (Supplemental Table 2A; site 1 and site 2) by luciferase reporter assay. miR-668 did not repress luciferase expression by the plasmid with site 1 sequence, but it inhibited luciferase expression by the plasmid containing site 2 (Figure 7F), suggesting that miR-668 may directly target site 2 to repress MTP18.

MTP18 promotes mitochondrial fragmentation and apoptosis in renal tubular cells. MTP18 has been implicated in mitochondrial fission and apoptosis $(16,17,47)$. To verify the role of MTP18 in renal tubular cells, we examined the effects of MTP18 knockdown in RPTCs. RPTCs were stably transfected with MTP18-specific shRNA, resulting in approximately $70 \%$ knockdown (Figure 8A). Due to the stress of transfection and puromycin selection, the stable cell lines showed high levels of mitochondrial fragmentation (Figure 8B). Nonetheless, mitochondrial fragmentation was reduced in MTP18-knockdown cells (Figure 8B), supporting a role of MTP18 in mitochondrial fission. MTP18-knockdown cells 
A

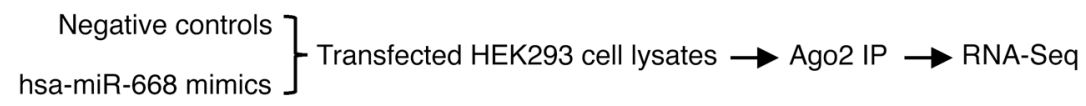
IP miR-668/IP NC>2 $\left\{\begin{array}{l}273 \text { pseudogene, noncoding RNA, unknown genes } \\ 407 \text { protein-coding genes }\left\{\begin{array}{l}124 \text { genes with predicted miR-668 targeting sites } \\ 283 \text { genes without miR- } 668 \text { targeting site }\end{array}\right.\end{array}\right.$

B

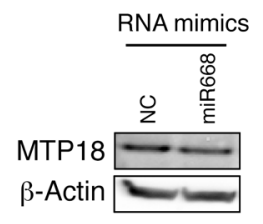

D
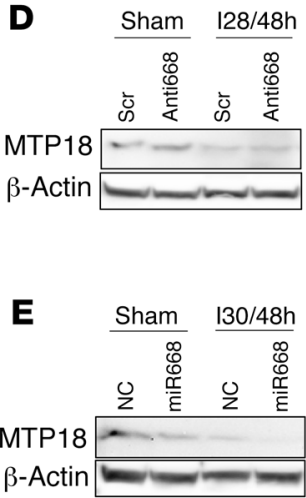

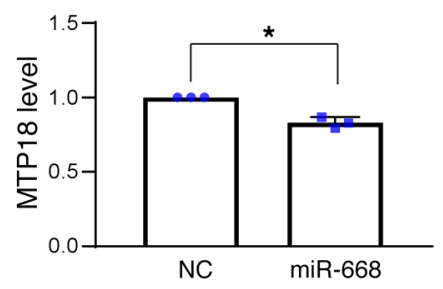

Sham

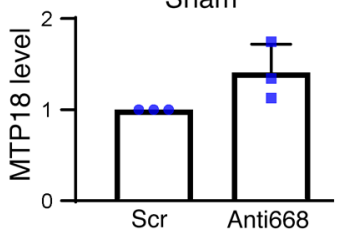

Sham

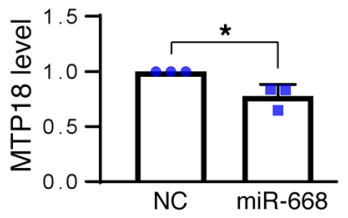

C

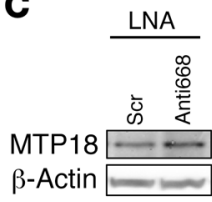

I28/48h

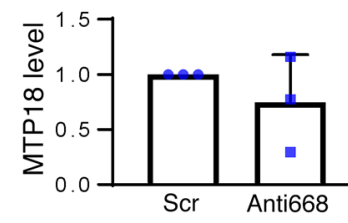

130/48h

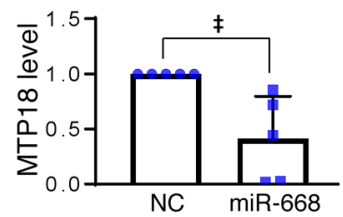

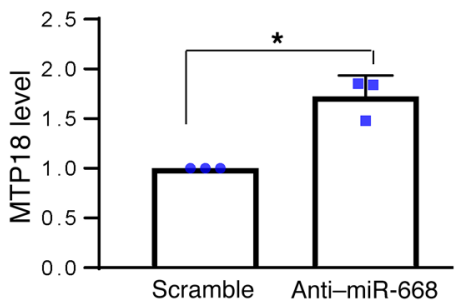

F

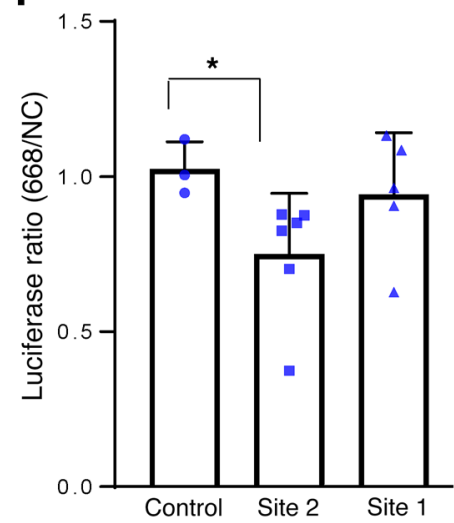

Figure 7. miR-668 targets MTP18 in ischemic AKI and renal tubular cells. (A) Schematic diagram depicting the procedure of Ago-2 immunoprecipitation of miRISC followed by RNA deep sequencing (RNA-Seq). HEK293 cells were transfected with negative control and miR-668 mimics. The miRISC complexes were pulled down with specific Ago-2 antibodies. The total RNA from HEK293 cells and the RNA from Ago-2 immunoprecipitates were subjected to RNASeq. (B) Representative immunoblots and densitometry analysis $\left(n=3 ;{ }^{*} P=0.0083\right.$, 1-tailed Student's $t$ test) of MTP18 showing that miR-668 mimic (MiR668) suppressed MTP18 expression in HEK293 cells as compared with negative control (NC) oligonucleotide. (C) Representative immunoblots and densitometry analysis ( $n=3$; ${ }^{*} P=0.0135$, 1-tailed Student's $t$ test) of MTP18 in HEK293 cells showing that anti-miR-668 LNA (Anti668) increased MTP18 expression as compared with scrambled LNA (Scr). (D) Representative immunoblots and densitometry analysis $(n=3)$ of MTP18 in mouse kidneys. Ischemic AKI (I28/48h) induced the decrease of MTP, which was prevented by anti-miR-668 LNA (Anti668). (E) Representative immunoblots and densitometry analysis of MTP18 in mouse kidneys. In comparison with negative control (NC) oligonucleotides, miR-668 mimic (MiR668) suppressed MTP expression in both sham-operated and ischemic AKI (I30/48h) mice ( $n=3$ for sham, ${ }^{*} P=0.0357 ; n=5$ for $130 / 48 \mathrm{~h},{ }^{\ddagger} P=0.0136 ; 1$-tailed Student's $t$ test). In the blots throughout this figure, $\beta$-actin was used as loading control for normalization. (F) Luciferase microRNA target reporter assay showing that miR-668 targets site 2 in the $3^{\prime}$-UTR of MTP18 ( $n=3$ for null control, $n=6$ for site $2, n=5$ for site $1 ;{ }^{*} P=0.0484,1$-way ANOVA with Dunn's multiple-comparisons test).

were also resistant to ATP depletion-induced apoptosis. In morphological analysis, azide-induced ATP depletion led to $26.7 \% \pm$ $2.4 \%$ apoptosis in nontarget shRNA-transfected cells, which was suppressed to $13.3 \% \pm 2.4 \%$ in MTP18-knockdown cells (Figure 8, $\mathrm{C}$ and D). Consistently, MTP18-knockdown cells had significantly lower caspase activity following azide treatment as compared with nontarget shRNA-transfected cells (Figure 8E).

The protective effect of miR-668 is lost in MTP18-deficient renal tubular cells. To determine whether miR-668 protects renal tubular cells via MTP18, we compared the effects of miR-668 in MTP18 shRNA knockdown cells versus nontarget shRNA-transfected cells. Without azide injury, all cells showed low, basal levels of apoptosis (Figure 9, A-C). Following azide-induced ATP depletion, nontarget shRNA cells transfected with negative control oligonucleotides showed $29 \%$ apoptosis, which was suppressed to approximately $15 \%$ by miR-668, verifying the protective effect of this microRNA (Figure
9B). In MTP18-knockdown cells, azide treatment induced approximately 17\% apoptosis, and notably, miR-668 did not attenuate apoptosis (Figure 9B). Consistently, miR-668 suppressed caspase activation during azide treatment in nontarget shRNA-transfected cells, but not in MTP18 shRNA knockdown cells (Figure 9C). Thus, the protective effect of miR-668 was lost in the absence of MTP18, suggesting that miR-668 protects cells via MTP18.

\section{Discussion}

In this study, we have demonstrated that miR-668 is induced via HIF-1 during ischemic AKI and, upon induction, miR-668 represses MTP18 for the maintenance of mitochondrial dynamics and the viability of kidney tubular cells. Notably, miR-668 induction has been verified not only in animal and cell models but also in the samples from 2 sets of AKI patients (Figure 1). The first set of patients were diagnosed with AKI, but the etiology or cause of 


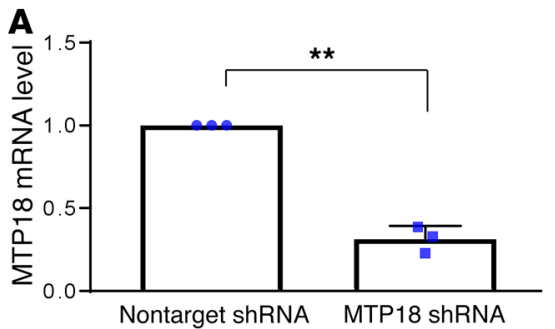

C
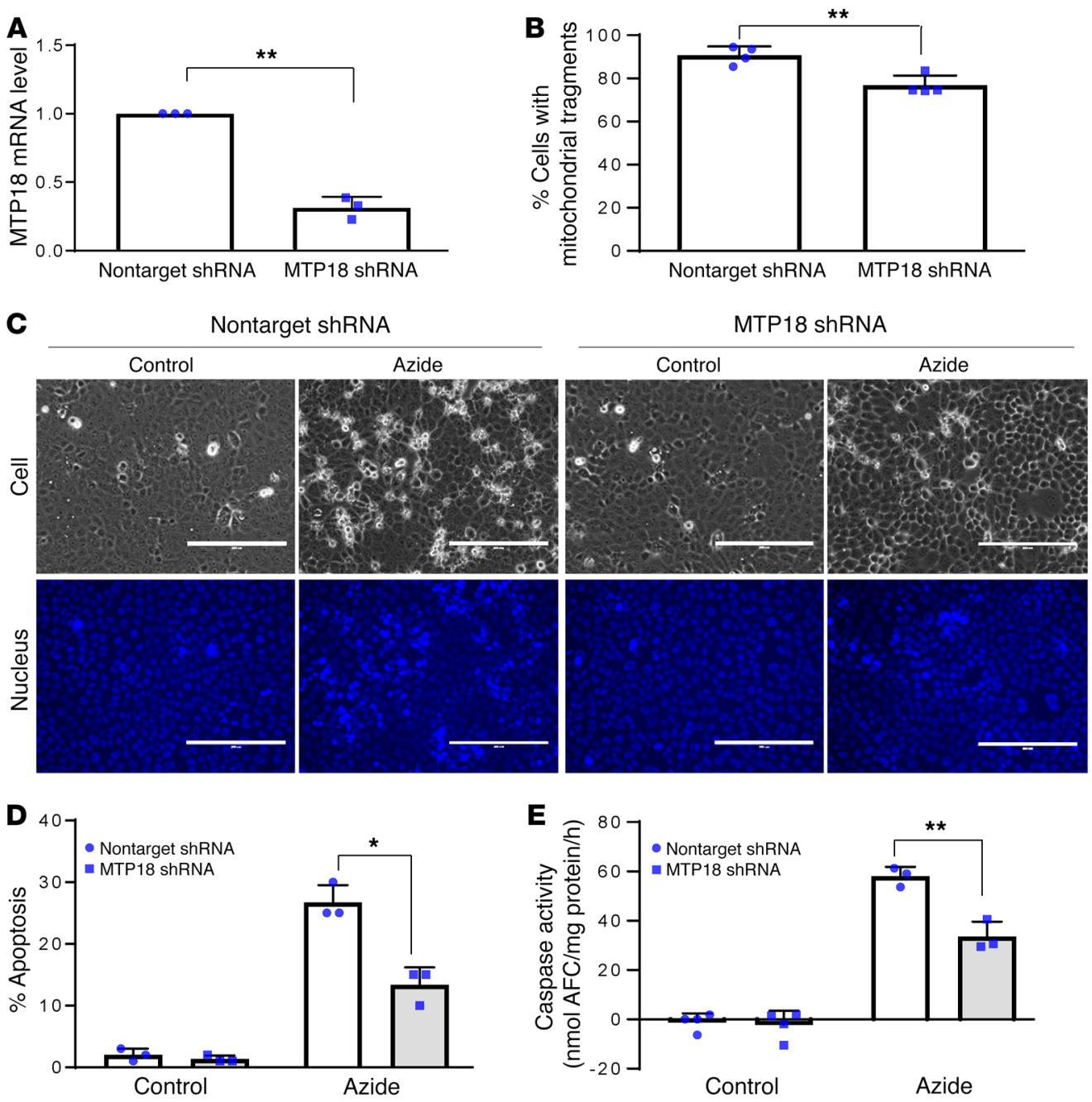

Figure 8. MTP18 promotes mitochondrial fragmentation and apoptosis in renal tubular cells. RPTCs were stably transfected with nontarget or MTP18 shRNA, and then treated with azide for 2.25 hours to induce ATP depletion/ repletion injury or left untreated as control. (A) qPCR analysis to verify the silencing effect of MTP18 shRNA after stable transfection ( $n=3$; ${ }^{* *} P=0.0044$, 2-tailed Student's $t$ test). (B) Percentage of cells with fragmented mitochondria to show that knockdown of MTP18 reduced mitochondrial fragmentation $\left(n=4 ;{ }^{* *} P=0.004\right.$ 2-tailed Student's $t$ test). (C) Representative images of RPTCs to show that knockdown of MTP18 protected against apoptosis. Scale bar: $0.2 \mathrm{~mm}$. (D) Percentage of apoptosis by counting of the cells with typical apoptotic morphology ( $n=3$; ${ }^{*} P=0.0048,2$-tailed Student's $t$ test). (E) Caspase activity measured by enzymatic assay ( $n$ $=4$ for control groups, $n=3$ for azide groups; ${ }^{* *} P=0.007$, 2-tailed Student's $t$ test).
AKI was unclear. The kidney biopsies from these patients had significantly higher levels of miR-668 as compared with the biopsies of non-AKI patients. Notably, serum creatinine and BUN, 2 clinical indices of AKI, demonstrated a good correlation with the level of miR-668 expression, further confirming miR-668 induction in kidneys in general AKI patients. The second set of patients were from cardiac surgery with cardiopulmonary bypass, and therefore their AKI had a defined etiology of renal ischemia/reperfusion due to aortic cross-clamping and subsequent release. Ethical considerations prevented us from obtaining kidney biopsies from these patients. However, in urine samples we detected significantly higher levels of miR-668 in the patients developing AKI after surgery than in the patients without AKI (Figure 1E). Interestingly, serum samples from AKI and non-AKI patients after surgery had similar levels of miR-668 (Figure 1D), suggesting that miR-668 detected in the urine of AKI patients was mainly produced by kidney tissues. Together, these results demonstrate miR-668 induction in AKI in human patients. Our cell and mouse model studies have further verified miR-668 induction in kidney proximal tubular cells during hypoxia in vitro and renal ischemia/reperfusion and UUO in vivo in mice (Figure $1, \mathrm{~F}-\mathrm{H}$ ).

Mechanistically, we have identified HIF-1 as a key upstream factor for miR-668 induction during ischemic AKI (Figure 2). In cultured cells, miR-668 induction by hypoxia was completely suppressed in HIF- $1 \alpha$-null cells. Moreover, miR-668 induction during ischemic AKI in mice was abrogated by specific ablation of HIF-1 from kidney proximal tubules. We further verified the binding of HIF-1 to a predicted hypoxia response element in the promoter region of the miR-668 gene, providing the evidence for direct regulation of miR- 668 by HIF-1. HIFs are the "master" regulators of gene expression during hypoxia/ischemia (42). In kidneys, HIF-1 is induced mainly in renal tubule cells during ischemic AKI, whereas HIF-2 is expressed by endothelium $(44,48)$. We and others recently demonstrated an important role of HIF-1 in the induction of multiple microRNA species during ischemic AKI, including miR-687, miR-489, and miR-21 $(32,36,40)$. The present study further suggests HIF-1-mediated induction of miR-668. Interestingly, while miR-489, miR-21, and miR-668 were shown to be protective, miR-687 appeared to promote kidney injury $(32,36,40)$. Thus, although HIF-1 is generally recognized for its protective or adaptive effects, it may mediate the expression of both protective and injury genes. As such, it is important to identify the specific genes regulated by HIF for specific and effective therapy $(8,49)$.

Functionally, we have demonstrated that miR-668 induction is a protective response in renal tubular cells. This was initially suggested by the localization of miR-668 in proximal tubules that 
A

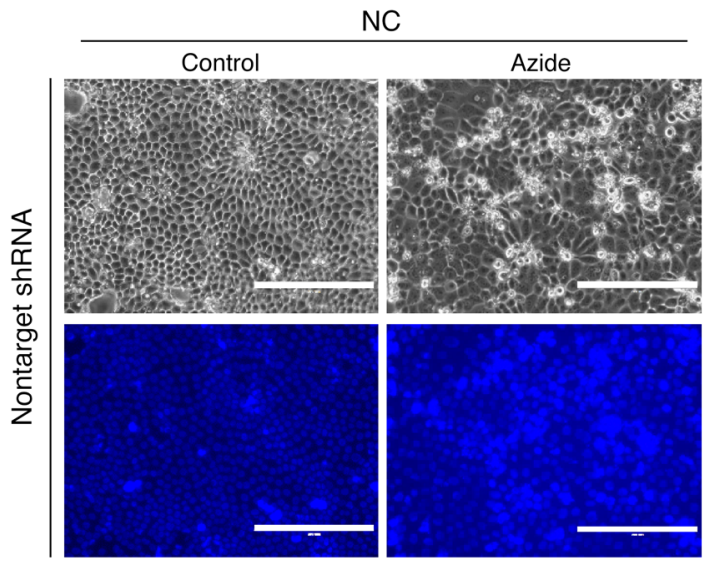

NC

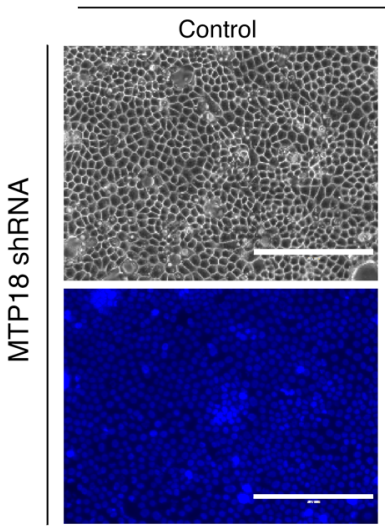

B
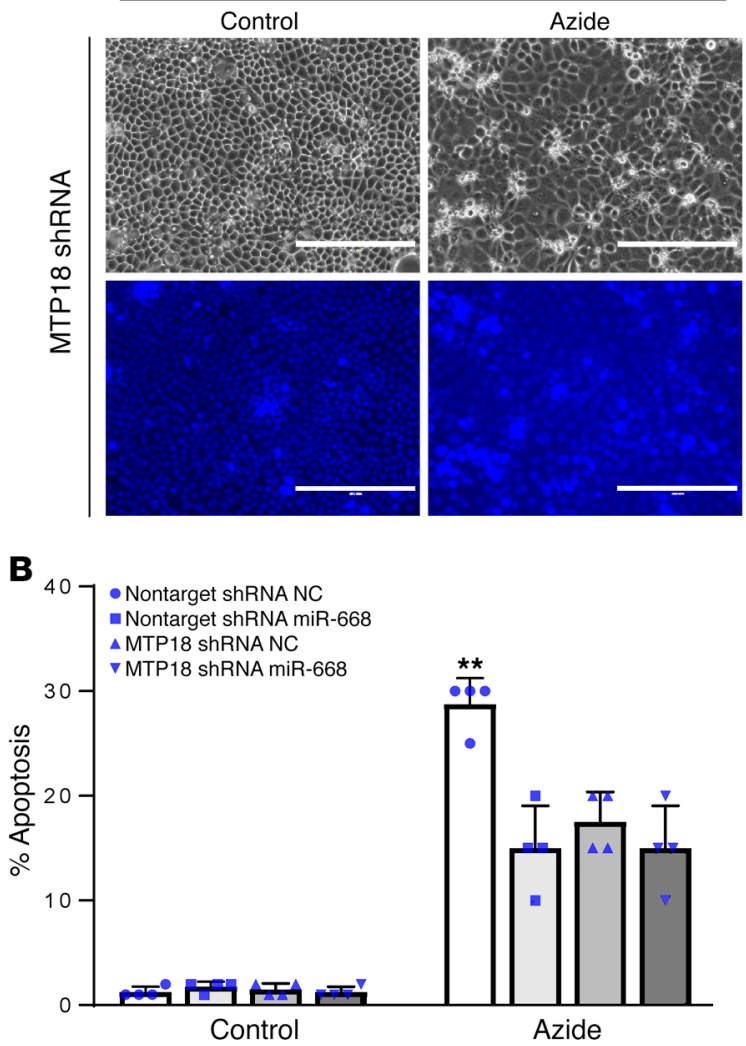

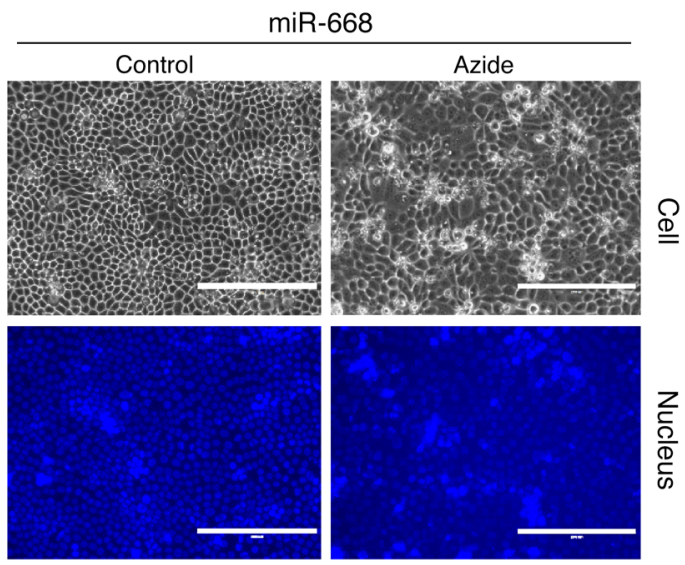

$\stackrel{\circledast}{=}$
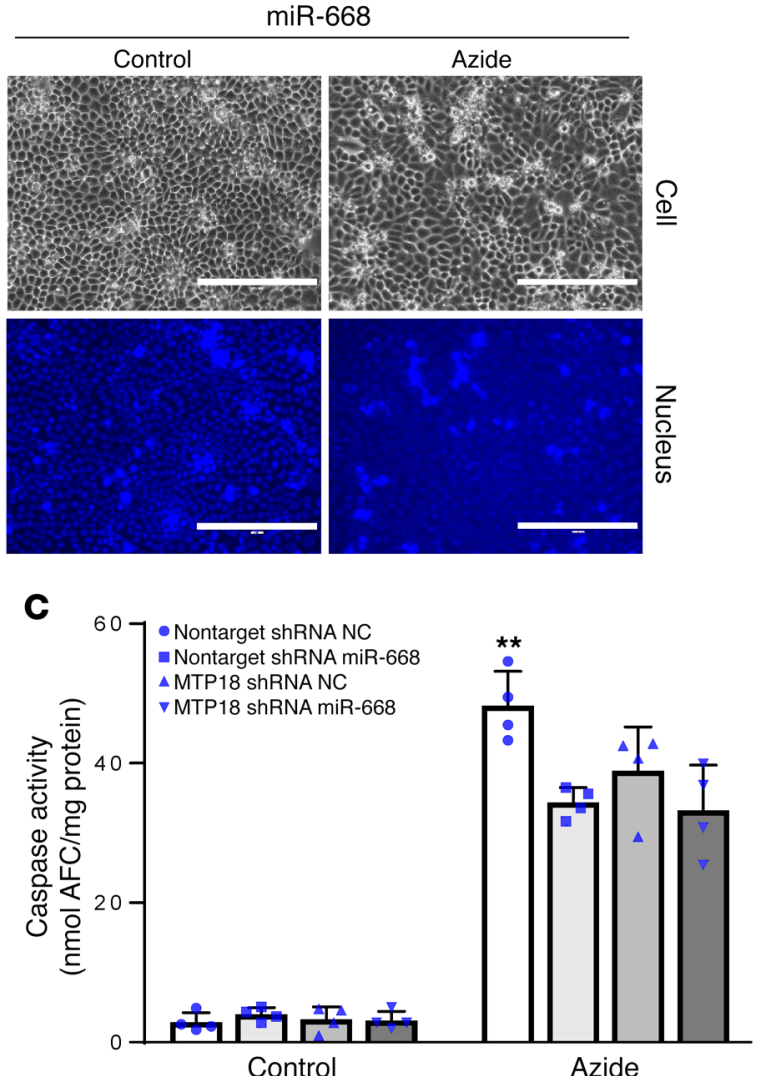

Figure 9. The protective effect of miR-668 is lost in MTP18-knockdown renal tubular cells. RPTCs stably transfected with MTP18 shRNA or nontarget oligonucleotides were transfected with negative control (NC) or miR-668 mimics. The cells were then treated with azide-induced ATP depletion or left untreated as control. (A) Representative cell and nucleus images. Scale bar: $0.2 \mathrm{~mm}$. (B) Percentage of apoptosis by counting of the cells with typical apoptotic morphology $\left(n=4 ;{ }^{* *} P=0.0003\right.$, 1-way ANOVA). (C) Caspase activity $\left(n=4 ;{ }^{* *} P=0.0066,1\right.$-way ANOVA). The results show that miR-668 suppressed azide-induced apoptosis in nontarget shRNA-transfected cells, but not in MTP18-knockdown cells.

were relatively intact in structure during ischemic AKI (Figure $1 \mathrm{H})$. We further showed that anti-miR-668 induced cell death in RPTCs, whereas miR-668 mimic protected against ATP depletion-associated apoptosis (Figure 3). Moreover, miR-668 mimic showed protective effects against ischemic AKI, whereas antimiR-668 exaggerated kidney injury in mice (Figures 4 and 5). Thus, both in vitro and in vivo data support a protective role of miR-668 in renal tubular cells. The cell biological function of miR668 is largely unknown. Nonetheless, the latest work by Luo et al. suggested the involvement of miR-668 in the radioresistance of breast cancer cells (41), an observation that is in line with our data for a cytoprotective function of this microRNA.

For in vivo study, anti-miR-668 LNA or miR-668 mimics were systemically administered to mice. Thus, we cannot exclude the possible off-target effects in other organs. Nonetheless, it is noteworthy that LNAs mainly accumulate in liver and kidney (50). In addition, anti-miR-668 LNA or miR-668 mimics did not induce obvious changes in animal behavior, renal function, and histology. 
In our study, we detected different levels of miR-668 expression in various organs, showing the highest expression in the heart, muscle, and kidney (data not shown). In kidneys, we observed that miR-668 exerted protection mainly by affecting renal apoptosis. In many previous studies, renal protective effects were shown by the reduction of both apoptotic and necrotic renal damage $(4,24$, 51). However, under specific conditions, the effect may be limited to one form of tubular cell death. For example, global or proximal tubule-specific knockout of Bak only suppressed tubular apoptosis (46). Thus, it is possible that mainly renal apoptosis is regulated and functionally accounts for the effect on AKI in certain conditions.

How does miR- 668 protect? In this regard, we made the novel observation that miR-668 suppressed mitochondrial fragmentation during hypoxia stress in RPTCs, whereas anti-miR-668 promoted mitochondrial fragmentation (Figure 6). Mitochondrial fragmentation, as a result of the disruption or perturbation of mitochondrial dynamics, is a critical pathogenic event in kidney tubular damage in acute as well as chronic kidney diseases $(4,6$, 7,11-13). Therefore, our results suggest that miR-668 may protect kidney tubular cells during ischemic AKI by preserving mitochondrial dynamics and integrity for cell survival.

Mitochondrial damage is an early event in ischemic AKI. In our previous study, we detected mitochondrial fragmentation at 15 minutes of reperfusion after kidney ischemia $(4,46)$. However, there is a delay for renal function measurements (BUN and creatinine) to reflect the injury (52). In addition, the severity of initial injury also determines the recovery of kidney $(53,54)$. The presence of earlier recovery in the scramble group also indicates less initial injury. Furthermore, miR-668 may also protect kidneys in the subacute injury phase. miR-668 mainly regulates renal apoptosis (Figures 4 and 5), whereas apoptosis may result from both ischemia and the injury factors accumulated during reperfusion period (55). In animal models of ischemic AKI, apoptosis is initially detected at 12-24 hours after ischemia and may maintain at a certain level or increase because of the severity of injury (56, 57). Thus, the sustained inhibition of miR-668 after ischemia may time-dependently augment apoptosis and renal injury. Finally, it is still unknown whether miR-668 facilitates kidney recovery by regulating other pathways, such as tubular repair and renal fibrosis.

The key to elucidating the pathophysiological role of a microRNA is to identify the downstream target gene(s). In this study, we have examined the targets of miR-668 using the nonbiased method of miRISC immunoprecipitation followed by RNA deep sequencing. This analysis led to the identification of 124 protein-coding gene mRNAs that had miR-668 targeting sequences and increased accumulation in miRISC upon miR-668 treatment. Therefore, these 124 genes are likely real targets of miR-668. To identify the target genes accounting for the observed effect of miR-668 on mitochondrial dynamics, we further conducted bioinformatics analysis and literature search. Interestingly, of these 124 genes, MTP18 is the only one that has been implicated in mitochondrial dynamics. MTP18 is a PI3K-dependent protein that is localized in mitochondria and has been shown to promote mitochondrial fission $(16,17)$. Consistently, in our study, knockdown of MTP18 prevented mitochondrial fragmentation in RPTCs (Figure 8). In cells, miR-668 mimic decreased MTP18 expression, whereas anti-miR-668 LNA increased MTP18 expression (Figure 7, B and C). In mice, miR-668 mimic suppressed MTP18 expression in kidneys in both sham-operated control mice and ischemic AKI mice (Figure 7E). Moreover, in luciferase microRNA target reporter assay, miR- 668 repressed MTP18 by binding to at least 1 of the predicted target sites at the 3'-UTR of the MTP18 gene (Figure $7 F)$. Collectively, these results provide convincing evidence that MTP18 is a direct target of miR-668 and, by repressing MTP18, miR-668 may preserve mitochondrial dynamics and prevent mitochondrial fragmentation for tubular cell survival in ischemic AKI. Thus, although miR-668 may target other genes, our data indicate that MTP18 is the major target that mediates the effects of miR-668 on mitochondria in ischemic AKI.

Nonetheless, we cannot exclude the indirect effects of other potential targets from the regulation of mitochondrial dynamics. First, other microRNAs accumulated in miRISC when miR-668 was overexpressed, and some of these microRNAs may also target MTP18. For example, the deep sequencing data (GEO GSE117805) indicate the accumulation of MIR17HG (miR-17-92 cluster) in miRISC. Among them, miR-18a is also predicted to target MTP18 by TargetScan. In the luciferase microRNA target reporter assay, miR668 showed a moderate (25\%) effect on MTP18 3'-UTR sequence (Figure 7F). Similarly, luciferase assay detected about 30\% inhibition by miR-375 on its target HNF-1 $1 \beta$ sequence in our recent study (58). The luciferase assay may be affected by multiple factors such as the transfection and expression efficiency of the microRNAs and luciferase constructs, and the 3D RNA structure of 3'-UTR due to the DNA sequence chosen to insert into the luciferase construct $(59,60)$. Regardless, we cannot exclude the possible effects of other microRNAs. Second, despite our evidence that MTP18 is mainly responsible for the protective effect of miR-668 (Figure 9), miR-668 may target other genes to regulate mitochondrial dynamics. For instance, there are $3 \mathrm{TNF}$ superfamily/receptor proteins (TNFSF4, TNFRSF8, TNFSF10) that are related to cell death regulation. Among them, TNFSF10 (TRAIL) has been reported to promote mitochondrial fission by regulation of Drp1 phosphorylation status (61), though we did not detect obvious Drp1 (S637) change in our study (Figure 6E). Finally, it is also possible that software prediction cannot reveal all the potential targets of miR-668. Currently all microRNA target prediction softwares are based on the examination of 3'-UTR, the canonical sites for microRNAs to repress target gene expression. But recent studies suggest that microRNAs may also regulate target gene expression by target 5 '-UTR or coding region $(62,63)$. This is also one of the possible reasons why many genes revealed in Ago-2 immunoprecipitation assay do not have miR-668 binding sites at 3'-UTR.

In cultured renal tubular cells, miR-668 mimic attenuated MTP18 expression and anti-miR-668 induced MTP18, while miR668 mimic suppressed MTP18 in mouse kidneys (Figure 7, B, C, and E), confirming MTP18 as a target gene of miR-668. However, anti-miR-668 only had a minor effect on MTP18 in mice (Figure 7D). Several possibilities may account for the lesser efficiency of anti-miR-668 in vivo. First, the efficiency of anti-miR-668 in vivo delivery may not be high enough. To support this, qPCR analysis (Figure 4B) showed only a marginal inhibition of miR-668 by anti-miR-668 in sham control condition. In ischemic condition, anti-miR-668 showed a better inhibitory effect (40\%), but the inhibition was not complete (Figure 4B). Second, besides miR- 
668, MTP18 may also be inhibited by other pathways, especially in ischemic condition. In that case, the inhibition of MTP18 in ischemic AKI cannot be totally reversed by anti-miR-668. On the contrary, when miR-668 was overexpressed in mice, it caused about $20 \%$ inhibition of MTP18 in sham control and $60 \%$ inhibition in ischemic condition ( 60\%) (Figure 7E).

Compared with other mitochondrial fission proteins (e.g., Drp1, Fis-1), MTP18 is much less studied. While the pro-fission function of MTP18 is established, its role in cell death regulation remains controversial. In cardiomyocytes and gastric cancer cells, MTP18 knockdown inhibited apoptosis, suggesting that MTP18 may promote cell death by inducing mitochondrial fission and fragmentation $(47,64)$. However, in human HaCaT keratinocytes, MTP18 knockdown induced cell death and sensitized the cells to apoptotic stimuli, even though MTP18 was shown to promote mitochondrial fission in these cells (17). Therefore, the function of MTP18 may be cell type and context dependent, and, in addition to mitochondrial fission, MTP18 may have other pathways to regulate cell death. In RPTCs, we show that knockdown of MTP18 led to a reduction of mitochondrial fragmentation, verifying the role of MTP18 in mitochondrial fission. Moreover, knockdown of MTP18 protected cells from ATP depletion-induced apoptosis, suggesting that MTP18 is pro-death in renal tubular cells (Figure 9). Thus, MTP18 may contribute to ischemic AKI by promoting mitochondrial fragmentation with consequent mitochondrial damage and tubular cell death.

In conclusion, this study has demonstrated miR-668 induction in ischemic AKI in human patients as well as cell and animal models. miR-668 induction is mediated by HIF- 1 in kidney proximal tubules, and, upon induction, miR-668 targets or represses the mitochondrial fission protein MTP18 to preserve mitochondrial dynamics and prevent mitochondrial fragmentation for tubular cell survival (Supplemental Figure 8). Discovery of the pathway of HIF-1/miR-668/MTP18 not only provides significant new insights into the understanding of ischemic AKI but also unveils new therapeutic targets and strategies.

\section{Methods}

Human patient participants and sample collection. For general AKI, patients were recruited in the Department of Nephrology at Shanghai Changzheng Hospital of the Second Military Medical University in China. Kidney biopsy, urine, and whole-blood samples were collected for diagnosis, and remaining samples were extracted for total RNA and miR-668 qPCR analysis. The patients with cardiopulmonary surgery were recruited in the Department of Cardiothoracic Surgery at Shanghai Changhai Hospital of Second Military Medical University in China. These patients experienced surgery with cardiopulmonary bypass and aortic cross-clamping operations. Urine and blood samples were collected before surgery or $0,3,9$, or 24 hours after surgery for total RNA extraction and miR-668 qPCR analysis. All human samples were collected with the patient's consent following the Institution Human Sample Collection Regulation of the Second Military Medical University in China.

Animals and ischemic AKI induction. C57BL/6J and Hif- $1 \alpha^{\text {tm3Rsjo }}$ floxed mice were originally from The Jackson Laboratory. PEPCK-Cre mice were originally from Volker Haase at Vanderbilt University (Nashville, Tennessee, USA) (65). Hif- $1 \alpha^{\text {tm3Rsjo }}$ floxed mice were crossed with PEPCK-Cre mice to obtain the WT and PT-HIF-1 $\alpha$-knockout mice as described recently (36). Ischemic AKI in mice was induced by bilateral renal ischemia/reperfusion with 8- to 10-week-old male mice as described before (66). Briefly, mice were anesthetized with $60 \mathrm{mg} / \mathrm{kg}$ pentobarbital and kept on a heating blanket with the body temperature maintained at $36.5^{\circ} \mathrm{C}$. The kidney pedicles were exposed by flank incision and clamped with microvessel clips to block the blood flow to kidney. The clips were released at defined ischemic time for reperfusion. Sham operation had the same procedure but without clipping of the kidney pedicles.

Cell culture models. RPTCs were originally from U. Hopfer at Case Western Reserve University (Cleveland, Ohio, USA) and were cultured as described before (67). HEK293 cells were originally purchased from ATCC. LNA oligonucleotides, microRNA mimic oligonucleotides, siRNA oligonucleotides, and DNA plasmids were transiently transfected into RPTCs or HEK293 cells with Lipofectamine 2000 (Life Technologies) following the manufacturer's instructions. To inhibit miR-668, $200 \mathrm{nM}$ anti-miR-668 LNAs (Exiqon Inc.) were transfected into cells, while scrambled-sequence LNA oligonucleotides were transfected as negative control. To upregulate miR-668, $200 \mathrm{nM}$ miR668 mimics (Life Technologies) were transfected in cells, while the negative control oligonucleotides were transfected as control. To label mitochondria, $0.2 \mu \mathrm{g}$ pDsRed2-Mito (Mito-Red, Clontech) was transiently transfected into cells.

WT and HIF- $1 \alpha$-knockout mouse embryonic fibroblasts (MEFs) were originally from Gregg Semenza at Johns Hopkins University (Baltimore, Maryland, USA). The cells were seeded in culture dishes to reach full confluence the next day for experiment. For hypoxia treatment, the cell culture medium was prebalanced in $1 \% \mathrm{O}_{2}$ overnight, and then the cells were incubated in the hypoxia-balanced medium and kept in a hypoxia $\left(1 \% \mathrm{O}_{2}\right)$ chamber. As control, another group of cells were kept in a regular cell culture incubator with $21 \% \mathrm{O}_{2}$.

MTP18-knockdown cells were generated by stable transfection of MTP18 shRNA in RPTCs. Briefly, MISSION shRNAs specifically targeting MTP18 were packaged into lentivirus with MISSION Lentiviral Packaging Mix following the instruction of the manufacturer (Sigma-Aldrich). Then shRNAs were transduced to RPTCs by lentivirus infection, and the cells were selected with and maintained in media containing $2.5 \mu \mathrm{g} / \mathrm{ml}$ puromycin to have MTP18 stable knockdown cells. Nontarget MISSION shRNAs were used to generate transfection control cells for comparison. The MTP18 shRNA sequence was CCGGCTTCACCATCAACCGTCTGTGCTCGAGCACAGACGGTTGATGGTGAAGTTTTTG. To confirm MTP18 knockdown, total RNA of RPTC cells was collected and reverse-transcribed with iScript cDNA Synthesis Kit from Bio-Rad for quantitative real-time PCR using primers predesigned by Integrated DNA Technologies Inc. The primers for rat MTP18 mRNA were: forward, GCCATGTTCTTTGATGTGAGTG; and reverse, CTGTCATTGTCCACCCCATT; and for 18S rRNA as internal control: forward, CTACCACATCCAAGGAAGCA; and reverse, TTTTTCGTCACTACCTCCCCG.

To induce apoptosis, the cells were incubated in glucose-free Krebs-Ringer bicarbonate solution with $10 \mathrm{mM}$ sodium azide for 3 hours to induce ATP depletion followed by 2 hours of recovery in full culture medium (4). In the study of MTP18 stable knockdown cells, the cells were treated with azide for ATP depletion for 2 hours and 15 minutes because of the injury sensitivity of these cells.

In situ hybridization of miR-668. In situ hybridization was performed as described before (36). Briefly, perfusion-fixed kidney samples were balanced in $20 \%$ sucrose in PBS overnight in $4^{\circ} \mathrm{C}$. Fresh cryo- 
sections of kidneys were dried for 20 seconds in air and further fixed in $4 \%$ paraformaldehyde for 20 minutes in room temperature. After PBS wash, the specimens were treated with $1 \mu \mathrm{g} / \mathrm{ml}$ proteinase $\mathrm{K}$ for permeabilization. The expression of miR-668 was further detected with IsHyb in situ hybridization kit (Biochain Institute Inc.) following the manufacturer's instructions. Briefly, the slides were incubated with prehybridization solution at $78^{\circ} \mathrm{C}$ for 3 hours. The hybridization mixture was then prepared by mixing of digoxigenin-labeled LNA probe (Exiqon Inc.) and hybridization solution to detect mmu-miR-668. The hybridization mixture was heated at $65^{\circ} \mathrm{C}$ for 5 minutes and cooled on ice. After overnight hybridization at $73^{\circ} \mathrm{C}$, the specimens were washed with serial dilutions of buffer and incubated with anti-digoxigenin alkaline phosphataseconjugated antibody. The final signal was revealed by NBT/BCIP solution (Biochain Institute Inc) and analyzed by light microscopy.

ChIP. ChIP was conducted as described previously $(32,36)$ with minor modifications. Briefly, WT or HIF- $1 \alpha$-null MEFs were subjected to 6 hours of $1 \% \mathrm{O}_{2}$ or normoxia treatment. The cells were then fixed with $0.75 \%$ formaldehyde and then neutralized with glycine. The cells were collected and the chromatin samples were sonicated to shear the DNA. The DNA from 50- $\mu$ l aliquots of the sonicated chromatin samples was used as INPUT DNA. According to the INPUT DNA concentration, the chromatin samples were diluted with sonication buffer to $1 \mu \mathrm{g} / \mathrm{ml} \mathrm{DNA}$ and precleaned with $1 \mathrm{mg} / \mathrm{ml} \mathrm{BSA}$ and $40 \mu \mathrm{l}$ of protein A agarose beads (Santa Cruz Biotechnology) in $1 \mathrm{ml}$ reaction buffer. After 2 hours of incubation, the beads were removed, and $4 \mu \mathrm{g}$ of antiHIF-1 $\alpha$ antibody (Cayman Chemical, 10006421) was added to the reaction for overnight incubation to pull down the chromatin. Finally, the antibodies were precipitated with protein A agarose beads. After serial washes, the precipitated DNA fragments were eluted and purified. The precipitated DNA samples with specific HIF-1 binding sites were quantified by real-time PCR using INPUT DNA for normalization. The following primers were used for HIF-1 binding site detection: binding site 1, forward, CAGGGTGTGTGACTGGTTGA; reverse, TTCATCCTGGTCCACTGAGC; binding site 2, forward, TCCCATGAGGGACCTGAAGT; reverse, TGTGGTAGTGTGGCGATGTC.

In vivo delivery of anti-miR-668 LNA and miR-668 mimic. Anti-miR668 and control LNA oligonucleotides were dissolved in nuclease-free PBS, and administered to mice through 1 tail vein injection as described before $(32,36)$. miR- 668 and control RNA oligonucleotides were delivered to mice with Invivofectamine 2.0 (Life Technologies) through 1 tail vein injection following the manufacturer's instructions (38).

miRISC immunoprecipitation. RISC immunoprecipitation was performed as described in our previous study (36). Briefly, HEK293 cells were cotransfected with FLAG-Ago-2 plasmid (Addgene) or control plasmid (p3xFLAG-Myc-CMV-24 Expression Vector, Sigma-Aldrich) and $100 \mathrm{nM}$ scramble RNA oligonucleotides or hsa-miR-668 mimics (Dharmacon Research Inc.). Total RNA extraction and RISC immunoprecipitation were conducted after 48 hours of transfection. The RISCs were pulled down from cell lysate with anti-FLAG resin (Sigma-Aldrich, A2220), and the RNA samples from RISC immunoprecipitation products were extracted with mirVana miRNA Isolation Kit (Life Technologies).

RNA deep sequencing and data analysis. Three sets of RNA samples from HEK293 cells or miRISC immunoprecipitation products were subjected to RNA-Seq as described previously (68). Briefly, cDNA libraries were built with $5 \mu \mathrm{g}$ of RNA, followed by cluster generation and sequencing. The RNA-Seq data were analyzed by an in-house pipeline that included read mapping and alignment, transcript construc- tion, quantification of transcript abundance, and identification of differential expression, using the Bowtie (http://bowtie-bio.sourceforge. net/index.shtml), TopHat version 2 (http://tophat.cbcb.umd.edu/), and Cufflinks (http://cufflinks.cbcb.umd.edu/) databases. Differential expression of transcripts was identified using false discovery rate less than 0.05. The reads that showed a more than 2-fold increase in the hsa-miR-668 group compared with the negative control group in the precipitated RNA samples were considered to have potential miR668 binding activity. The deep sequencing data were uploaded to the NCBI's Gene Expression Omnibus (GEO) database (GEO GSE117805).

Quantitative real-time PCR. Total RNAs were extracted from kidney tissues and cells with mirVana miRNA Isolation Kit (Life Technologies), and from serum or urine samples with mirVana PARIS RNA and Native Protein Purification Kit (Life Technologies), following the manufacturer's instructions. The level of miR-668 was quantified by TaqMan microRNA assay (Life Technologies) as described before $(24,32,36)$. For kidney samples, snoRNA-202 was used for normalization. For urine and blood samples, an equal amount of exogenous cel-miR-39 per volume was added immediately before RNA extraction and used for normalization. Only those patients with valid qPCR readings for all time points (before surgery and 0, 3, 9, and 24 hours after surgery) were used for final data analysis. The quantification of DNA fragments pulled down by ChIP and MTP18 mRNA levels was performed with iTaq Universal SYBR Green Supermix from Bio-Rad.

Luciferase microRNA target reporter assay. The target reporter assay was done as before $(24,32,36)$. DNA sequences of 50 bp containing the predicted miR-668 binding sites in the 3'-UTR region of MTP18 or null control insert without the predicted sites were synthesized (Genscript; see below for sequences). The sequences were subcloned into the 3'-UTR of the luciferase gene in the pMIR-REPORT luciferase plasmid (Life Technologies) between HindIII and SpeI sites. The luciferase plasmids were cotransfected with pMIR-REPORT $\beta$-gal Control Plasmid (Life Technologies) and $200 \mathrm{nM}$ microRNA mimics (negative control vs. hsa-miR-668 mimics; Life Technologies) into HEK293 cells. The luciferase activity and $\beta$-galactosidase were examined with the Luciferase Assay System and $\beta$-galactosidase Enzyme Assay System, respectively (Promega). The luciferase activity was normalized with $\beta$-galactosidase activity. The ratio of the normalized value between miR-668 and the negative control mimic group was used for comparison. The following sequences were used as inserts in luciferase expression plasmids: TGAGCTGGACAGAAGCTTAGAGACAAAGGCTTCAAGAAGCAGTGG (Site 1); GTGGTATGGCTGAACAAGGAGCGGCAGACAAC TCAGGGAGAAACT (Site 2); GTGGTATGGCTGAACAAGCACCGCCAGACAACTCAGGGAGAAACT (null control).

Bioinformatics analysis. The potential HIF-1 binding sites of miR668 were analyzed with the JASPAR database (http://jaspar.genereg. net). The potential mRNA targets of miR-668 were analyzed with miRanda (69) and Segal Lab of Computational Biology online software (http://genie.weizmann.ac.il/pubs/mir07/mir07_prediction.html). The potential miR- 668 binding sites of MTP18 were analyzed with Segal Lab of Computational Biology online software.

Measurement of renal function. Blood samples collected from tail vein or from heart puncture at sacrifice were clotted at room temperature and centrifuged to collect serum. Blood urea nitrogen was measured with Urea Nitrogen Direct from Stanbio Laboratory following the manufacturer's instructions. Serum creatinine was measured based on Jaffe reaction. Briefly, reaction mixture of $\mathrm{NaOH}$ and picric 
acid was prepared freshly and warmed up at $37^{\circ} \mathrm{C}$. Two values of the absorbance of $510 \mathrm{~nm}$ were read with a spectrophotometer at $20 \mathrm{sec}-$ onds and 80 seconds after addition of serum samples to the mixture. The difference of these 2 values was used for the calculation of creatinine concentration based on a standard curve.

Renal histology and TUNEL assay. For histology, mouse kidneys were fixed with $4 \%$ paraformaldehyde and embedded with paraffin. Kidney sections of $4 \mu \mathrm{m}$ were subjected to a standard H\&E staining. The histology score was obtained by estimation of the percentage of injured renal tubules in a blinded manner $(36,46)$. For TUNEL assay, paraffin-embedded kidney tissue sections were stained with In Situ Cell Death Detection Kit (Roche Applied Science). The slides were examined with fluorescent microscopy, and the TUNEL-positive cells were counted from 10 randomly picked images for each specimen in the outer medulla and kidney cortex region.

Quantification of apoptosis in cell cultures. Apoptosis was assessed by cell morphology and caspase activity measurement. For morphological examination, the cells were fixed with $4 \%$ paraformaldehyde and then stained with Hoechst 33342. The cells were examined with phase contrast microscopy and fluorescent microscopy, and the apoptosis percentage was estimated for each dish. The activity of caspases was measured by an enzymatic assay as described before (4). Briefly, cytosol lysates were collected with a buffer containing $1 \%$ Triton $\mathrm{X}-100$. Twenty-five micrograms protein was used for enzymatic reactions to convert DEVD-AFC to free AFC. The fluorescence of free AFC was measured at excitation $360 \mathrm{~nm} / \mathrm{emission} 530 \mathrm{~nm}$ before and after 1-hour reaction with a GENios plate reader (Tecan US). The caspase activity was calculated based on the standard curve as the production of nanomoles free AFC/hour/mg protein.

Analysis of mitochondrial fragmentation. Cells transfected with Mito-Red were fixed with $4 \%$ paraformaldehyde for 30 minutes followed by PBS rinse. The nuclei were stained by Hoechst 33342 . Then the cells were mounted onto slides with Vectashield Antifade mounting medium (Vector Laboratories Inc.) for further examination with confocal fluorescent microscopy or regular fluorescent microscopy. To quantify mitochondrial fragmentation, 150-200 cells from 10 randomly selected areas were examined for each slide to calculate the percentage of cells with mitochondrial fragmentation $(4,19)$.

Immunoblot analysis. Kidney tissues and cultured cells were lysed in SDS lysis buffer (62.5 mM Tris-HCl, pH 6.8, 2\% SDS, 10\% glycerol) to determine protein concentration using a Pierce BCA Protein Assay Kit (Thermo Fisher Scientific). Equal amount of protein samples or equal volume of IP products from same amount of input protein were loaded for standard reducing SDS-polyacrylamide gel electrophoresis followed by transferring to PVDF membrane. After blocking in $5 \%$ milk, the blot was exposed to primary antibody in $4^{\circ} \mathrm{C}$ overnight, followed by HRP-conjugated secondary antibody incubation. Finally, the blot was incubated with SuperSignal West Pico Chemiluminescent Substrate (Thermo Fisher Scientific) to record signals by exposure to $\mathrm{x}$-ray film or scanning with MyECL Imager (Thermo Fisher Scientific). Primary antibodies used were anti-HIF-1 $\alpha$ (Cayman Chemical, 10006421; or R\&D Systems, MAB1636), anti- $\beta$-actin (Sigma-
Aldrich, A5441), anti-active caspase-3 (Cell Signaling Technology, 9664S), anti-cyclophilin B (Cell Signaling Technology, 43603S), antiDrp1 (BD Transduction Laboratories, 611113), anti-Bak (Millipore, 06-536), anti-OPA1 (BD Biosciences, 612606), anti-OMA1 (Abcam, ab104316), anti-p-Drp1 (S637) (Cell Signaling Technology, 4867S), anti-mitofusin 2 (Sigma-Aldrich, M6444), anti-mitofusin 1 (Abnova, H00055669-M04), and anti-MTP18 (Abcam, ab198217). See complete unedited blots in the supplemental material.

Statistics. Student's $t$ test was used to show the significant difference between 2 groups, and ANOVA was used for multigroup difference analysis. Dunn's multiple comparisons for 1-way ANOVA and Fisher's least significant difference (LSD) for 2-way ANOVA were used. $P$ less than 0.05 was considered significant. Data were expressed as mean \pm SD. GraphPad Prism 7 and Microsoft Excel 2012 were used for all calculations.

Study approval. All animal experiments were conducted in accordance with a protocol approved by the Institutional Animal Care and Use Committee of Charlie Norwood VA Medical Center. All human studies were approved by the Institution Human Sample Collection Regulation of the Second Military Medical University, China. Written informed consent from participants or their guardians was received.

\section{Author contributions}

QW contributed to study design, experiments, data analysis, and manuscript preparation. YL contributed to RNA-seq and related data analysis. PL contributed to RNA-seq, related data analysis, and loading data to GEO. HS contributed to collection of human patient samples and clinical data, miR-668 analysis in human samples. SS contributed to collection of human patient samples and clinical data. MJL contributed to examination of renal histology. JW contributed to experiments. ML contributed to data analysis for RNA-seq. YH, QSM, and NSN contributed to data analysis and manuscript preparation. CM contributed to study design and data analysis for human patient samples. ZD contributed to overall study design, data analysis and manuscript preparation.

\section{Acknowledgments}

We thank Gregg Semenza for providing the HIF-1a-null MEF cell line, and Volker Haase for providing the PEPCK-Cre mouse line. The work was supported partly by the grants from the American Heart Association, the Augusta University Pilot Study Research Program, the NIH, and the Department of Veterans Affairs of the United States. ZD is a Senior Research Career Scientist of the Department of Veterans Affairs.

Address correspondence to: Zheng Dong, Charlie Norwood VA Medical Center, 1459 Laney Walker Boulevard, Augusta, Georgia 30912, USA. Phone: 706.721.2825; Email: zdong@augusta. edu. Or to: Qingqing Wei, Department of Cellular Biology and Anatomy, Medical College of Georgia at Augusta University, 1459 Laney Walker Boulevard, Augusta, Georgia 30912, USA. Phone: 706.721.3551; Email: qwei@augusta.edu.
1. Bonventre JV, Yang L. Cellular pathophysiology of ischemic acute kidney injury. JClin Invest. 2011;121(11):4210-4221.

2. Eltzschig HK, Eckle T. Ischemia and reperfu- sion - from mechanism to translation. Nat Med. 2011;17(11):1391-1401.

3. Sharfuddin AA, Molitoris BA. Pathophysiology of ischemic acute kidney injury. Nat Rev Nephrol.
2011;7(4):189-200

4. Brooks C, Wei Q, Cho SG, Dong Z. Regulation of mitochondrial dynamics in acute kidney injury in cell culture and rodent models. JClin Invest. 
2009;119(5):1275-1285

5. Bhargava P, Schnellmann RG. Mitochondrial energetics in the kidney. Nat Rev Nephrol. 2017;13(10):629-646.

6. Morigi M, et al. Sirtuin 3-dependent mitochondrial dynamic improvements protect against acute kidney injury. J Clin Invest. 2015;125(2):715-726.

7. Tran M, et al. PGC-1 $\alpha$ promotes recovery after acute kidney injury during systemic inflammation in mice. JClin Invest. 2011;121(10):4003-4014.

8. Ong SB, Subrayan S, Lim SY, Yellon DM, Davidson SM, Hausenloy DJ. Inhibiting mitochondrial fission protects the heart against ischemia/reperfusion injury. Circulation. 2010;121(18):2012-2022.

9. Grohm J, et al. Inhibition of Drp1 provides neuroprotection in vitro and in vivo. Cell Death Differ. 2012;19(9):1446-1458.

10. Guo X, Disatnik MH, Monbureau M, Shamloo M, Mochly-Rosen D, Qi X. Inhibition of mitochondrial fragmentation diminishes Huntington's disease-associated neurodegeneration. JClin Invest. 2013;123(12):5371-5388.

11. Wang $\mathrm{W}$, et al. Mitochondrial fission triggered by hyperglycemia is mediated by ROCK1 activation in podocytes and endothelial cells. Cell Metab. 2012;15(2):186-200.

12. Zhan M, Usman IM, Sun L, Kanwar YS. Disruption of renal tubular mitochondrial quality control by Myo-inositol oxygenase in diabetic kidney disease. J Am Soc Nephrol. 2015;26(6):1304-1321.

13. Perry HM, et al. Dynamin-related protein 1 deficiency promotes recovery from AKI. J Am Soc Nephrol. 2018;29(1):194-206.

14. Chan DC. Fusion and fission: interlinked processes critical for mitochondrial health. Annu Rev Genet. 2012;46:265-287.

15. Youle RJ, van der Bliek AM. Mitochondrial fission, fusion, and stress. Science. 2012;337(6098):1062-1065.

16. Tondera D, Czauderna F, Paulick K, Schwarzer $\mathrm{R}$, Kaufmann J, Santel A. The mitochondrial protein MTP18 contributes to mitochondrial fission in mammalian cells. JCell Sci. 2005;118(pt 14):3049-3059.

17. Tondera D, et al. Knockdown of MTP18, a novel phosphatidylinositol 3-kinase-dependent protein, affects mitochondrial morphology and induces apoptosis. J Biol Chem. 2004;279(30):31544-31555.

18. Cho SG, Du Q, Huang S, Dong Z. Drp1 dephosphorylation in ATP depletion-induced mitochondrial injury and tubular cell apoptosis. Am J Physiol Renal Physiol. 2010;299(1):F199-F206.

19. Brooks C, et al. Bak regulates mitochondrial morphology and pathology during apoptosis by interacting with mitofusins. Proc Natl Acad Sci US A. 2007;104(28):11649-11654.

20. Xiao X, Hu Y, Quirós PM, Wei Q, López-Otín C, Dong Z. OMA1 mediates OPA1 proteolysis and mitochondrial fragmentation in experimental models of ischemic kidney injury. Am JPhysiol Renal Physiol. 2014;306(11):F1318-F1326.

21. Wang JX, et al. miR-499 regulates mitochondrial dynamics by targeting calcineurin and dynamin-related protein-1. Nat Med. 2011;17(1):71-78.

22. Wang K, et al. miR-484 regulates mitochondrial network through targeting Fis1. Nat Commun
2012;3:781.

23. Duroux-Richard I, et al. miR-125b controls monocyte adaptation to inflammation through mitochondrial metabolism and dynamics. Blood. 2016;128(26):3125-3136.

24. Wei Q, Bhatt K, He HZ, Mi QS, Haase VH, Dong $Z$. Targeted deletion of Dicer from proximal tubules protects against renal ischemia-reperfusion injury. J Am Soc Nephrol. 2010;21(5):756-761.

25. Godwin JG, Ge X, Stephan K, Jurisch A, Tullius $S G$, Iacomini J. Identification of a microRNA signature of renal ischemia reperfusion injury. Proc Natl Acad Sci U S A. 2010;107(32):14339-14344.

26. Aguado-Fraile E, et al. miR-127 protects proximal tubule cells against ischemia/reperfusion: identification of kinesin family member 3B as miR-127 target. PLoS One. 2012;7(9):e44305.

27. Lan YF, et al. MicroRNA-494 reduces ATF3 expression and promotes AKI. JAm Soc Nephrol. 2012;23(12):2012-2023.

28. Xu X, et al. Delayed ischemic preconditioning contributes to renal protection by upregulation of miR-21. Kidney Int. 2012;82(11):1167-1175.

29. Li YF, et al. MicroRNA-21 in the pathogenesis of acute kidney injury. Protein Cell. 2013;4(11):813-819.

30. Bijkerk R, et al. Hematopoietic microRNA-126 protects against renal ischemia/reperfusion injury by promoting vascular integrity. JAm Soc Nephrol. 2014;25(8):1710-1722.

31. Lorenzen JM, et al. MicroRNA-24 antagonism prevents renal ischemia reperfusion injury. J Am Soc Nephrol. 2014;25(12):2717-2729.

32. Bhatt K, et al. MicroRNA- 687 induced by hypoxiainducible factor- 1 targets phosphatase and tensin homolog in renal ischemia-reperfusion injury. JAm Soc Nephrol. 2015;26(7):1588-1596.

33. Liang S, Wang W, Gou X. MicroRNA 26a modulates regulatory $\mathrm{T}$ cells expansion and attenuates renal ischemia-reperfusion injury. Mol Immunol. 2015;65(2):321-327.

34. Ranganathan $P$, et al. MicroRNA-150 deletion in mice protects kidney from myocardial infarction-induced acute kidney injury. Am J Physiol Renal Physiol. 2015;309(6):F551-F558.

35. Viñas JL, et al. Transfer of microRNA-486-5p from human endothelial colony forming cellderived exosomes reduces ischemic kidney injury. Kidney Int. 2016;90(6):1238-1250.

36. Wei Q, et al. MicroRNA-489 induction by hypoxia-inducible factor-1 protects against ischemic kidney injury. J Am Soc Nephrol. 2016;27(9):2784-2796.

37. Amrouche L, et al. MicroRNA-146a in human and experimental ischemic AKI: CXCL8dependent mechanism of action. JAm Soc Nephrol. 2017;28(2):479-493.

38. Hao J, et al. Induction of microRNA-17-5p by p53 protects against renal ischemia-reperfusion injury by targeting death receptor 6 . Kidney Int . 2017;91(1):106-118.

39. Wilflingseder J, et al. miR-182-5p inhibition ameliorates ischemic acute kidney injury. Am J Pathol. 2017;187(1):70-79.

40. Jia $P$, et al. MicroRNA-21 is required for local and remote ischemic preconditioning in multiple organ protection against sepsis. Crit Care Med. 2017;45(7):e703-e710.
41. Luo M, Ding L, Li Q, Yao H. miR-668 enhances the radioresistance of human breast cancer cell by targeting I $\mathrm{KB} \alpha$. Breast $\mathrm{Cancer}$. 2017;24(5):673-682.

42. Semenza GL. Oxygen sensing, hypoxia-inducible factors, and disease pathophysiology. Annu Rev Pathol. 2014;9:47-71.

43. Semenza GL. Hypoxia-inducible factor 1 and cardiovascular disease. Anпи Rev Physiol. 2014;76:39-56.

44. Rosenberger C, et al. Expression of hypoxiainducible factor-1alpha and -2alpha in hypoxic and ischemic rat kidneys. J Am Soc Nephrol. 2002;13(7):1721-1732.

45. Zhan M, Brooks C, Liu F, Sun L, Dong Z. Mitochondrial dynamics: regulatory mechanisms and emerging role in renal pathophysiology. Kidney Int. 2013;83(4):568-581.

46. Wei Q, Dong G, Chen JK, Ramesh G, Dong Z. Bax and Bak have critical roles in ischemic acute kidney injury in global and proximal tubulespecific knockout mouse models. Kidney Int . 2013;84(1):138-148.

47. Aung LHH, Li R, Prabhakar BS, Maker AV, Li P. Mitochondrial protein 18 (MTP18) plays a pro-apoptotic role in chemotherapy-induced gastric cancer cell apoptosis. Oncotarget. 2017;8(34):56582-56597.

48. Kapitsinou PP, et al. Endothelial HIF-2 mediates protection and recovery from ischemic kidney injury. J Clin Invest. 2014;124(6):2396-2409.

49. Nangaku M, Inagi R, Mimura I, Tanaka T. Epigenetic changes induced by hypoxia-inducible factor: a long way still to go as a target for therapy? J Am Soc Nephrol. 2015;26(7):1478-1480.

50. Straarup EM, et al. Short locked nucleic acid antisense oligonucleotides potently reduce apolipoprotein B mRNA and serum cholesterol in mice and non-human primates. Nucleic Acids Res. 2010;38(20):7100-7111.

51. Wei Q, Alam MM, Wang MH, Yu F, Dong Z. Bid activation in kidney cells following ATP depletion in vitro and ischemia in vivo. Am J Physiol Renal Physiol. 2004;286(4):F803-F809.

52. Vaidya VS, Ferguson MA, Bonventre JV. Biomarkers of acute kidney injury. Annu Rev Pharmaco Toxicol. 2008;48:463-493.

53. Forni LG, et al. Renal recovery after acute kidney injury. Intensive Care Med. 2017;43(6):855-866.

54. Venkatachalam MA, Weinberg JM, Kriz W, Bidani AK. Failed tubule recovery, AKI-CKD transition, and kidney disease progression. J Am Soc Nephrol. 2015;26(8):1765-1776.

55. Havasi A, Borkan SC. Apoptosis and acute kidney injury. Kidney Int. 2011;80(1):29-40.

56. Oberbauer R, Schwarz C, Regele HM, Hansmann C, Meyer TW, Mayer G. Regulation of renal tubular cell apoptosis and proliferation after ischemic injury to a solitary kidney. J Lab Clin Med. 2001;138(5):343-351.

57. Supavekin S, Zhang W, Kucherlapati R, Kaskel FJ, Moore LC, Devarajan P. Differential gene expression following early renal ischemia/reperfusion. Kidney Int. 2003;63(5):1714-1724.

58. Hao J, et al. MicroRNA-375 is induced in cisplatin nephrotoxicity to repress hepatocyte nuclear factor 1- $\beta$. J Biol Chem. 2017;292(11):4571-4582.

59. Sun G, Rossi JJ. Problems associated with 
reporter assays in RNAi studies. RNA Biol. 2009;6(4):406-411.

60. Cottrell KA, Szczesny P, Djuranovic S. Translation efficiency is a determinant of the magnitude of miRNA-mediated repression. Sci Rep. 2017;7(1):14884.

61. Suzuki-Karasaki Y, Fujiwara K, Saito K, SuzukiKarasaki M, Ochiai T, Soma M. Distinct effects of TRAIL on the mitochondrial network in human cancer cells and normal cells: role of plasma membrane depolarization. Oncotarget. 2015;6(25):21572-21588.

62. Lytle JR, Yario TA, Steitz JA. Target mRNAs are repressed as efficiently by microRNA-binding sites in the 5' UTR as in the $3^{\prime}$ UTR. Proc Natl
Acad Sci U S A. 2007;104(23):9667-9672.

63. Hausser J, Syed AP, Bilen B, Zavolan M. Analysis of CDS-located miRNA target sites suggests that they can effectively inhibit translation. Genome Res. 2013;23(4):604-615.

64. Wang K, et al. Circular RNA mediates cardiomyocyte death via miRNA-dependent upregulation of MTP18 expression. Cell Death Differ. 2017;24(6):1111-1120.

65. Rankin EB, Tomaszewski JE, Haase VH. Renal cyst development in mice with conditional inactivation of the von Hippel-Lindau tumor suppressor. Cancer Res. 2006;66(5):2576-2583.

66. Wei Q, Yin XM, Wang MH, Dong Z. Bid deficiency ameliorates ischemic renal failure and delays animal death in C57BL/6 mice. Am J Physiol Renal Physiol. 2006;290(1):F35-F42.

67. Woost PG, et al. Immortalization and characterization of proximal tubule cells derived from kidneys of spontaneously hypertensive and normotensive rats. Kidney Int. 1996;50(1):125-134.

68. Liu Y, Liu P, Yang C, Cowley AW, Liang M. Base-resolution maps of 5-methylcytosine and 5-hydroxymethylcytosine in Dahl S rats: effect of salt and genomic sequence. Hypertension. 2014;63(4):827-838.

69. Betel D, Wilson M, Gabow A, Marks DS, Sander C. The microRNA.org resource: targets and expression. Nucleic Acids Res. 2008; 36(Database issue):D149-D153. 